Check for updates

Cite this: RSC Adv., 2018, 8, 15709

Received 27th February 2018 Accepted 17th April 2018

DOI: $10.1039 / c 8 r a 01707 g$

rsc.li/rsc-advances

\section{Novel compatibilized nylon-based ternary blends with polypropylene and poly(lactic acid): morphology evolution and rheological behaviour $\dagger$}

\begin{abstract}
Amandine Codou, ${ }^{a}$ Andrew Anstey, ${ }^{a}$ Manjusri Misra (D) ab and Amar K. Mohanty (D) *ab
In this paper, the interaction between nylon 6 (PA6), polypropylene (PP) and poly(lactic acid) (PLA) is reported. To improve the compatibility between these immiscible polymers, a reactive compatibilization approach was used through extrusion with maleic anhydride grafted polypropylene (PP-g-MA). To further improve the compatibility of the phases, PLA was selected as a semi-polar polymer and a low molecular weight was used to assure a good droplet dispersion. All the blends were twin-screw extruded in the melt at different compositions. The morphologies of binary and ternary blends were investigated using microscopic techniques by means of scanning electron microscopy (SEM) and atomic force microscopy (AFM). The PP-g-MA grafting process was observed to have a dramatic effect on the compatibility of both the binary and ternary blends and while a drastic reduction of the PP dispersed phases particle size was observed, the affinity between PLA and PP was also highlighted. The surface tension of the homopolymers and the interfacial tension of the pairs of polymers were measured to characterize the interaction at their interfaces. The interaction of PA6/PLA appeared preferable to PLA/ PP, explaining the thinner dispersion obtained for PLA phase. The morphologies observed were compared to the predictions of spreading coefficient and minimum free energy models. While both models predicted the encapsulation of PP by the PLA phase, disagreeing with the morphological results, the rheological measurement gave an explanation for this phase separation. Using rheology measurements, the interaction between the phases was further investigated and the viscosity ratios were measured for the different pairs of polymers, stressing the high interaction between PA6 and PLA with and without compatibilizer. The droplet size of the dispersed phases appeared to substantially influence the chain relaxations in the melt.
\end{abstract}

\section{Introduction}

Polyamides are among the most widely used engineering thermoplastics, with a wide range of applications due to their high strength and resistance to chemicals and abrasion. Polyamide blends with commodity polymers such as polypropylene (PP), polyethylene (PE), and polystyrene (PS) have been investigated for many years. Blending with these polymers is intended to improve attributes such as moisture absorption, processability, and cost compared to neat nylon. Manipulation of blending ratios allows the tailoring of these parameters, as well as strength and stiffness, for different applications as necessary. Polyamide blends with polyolefins

\footnotetext{
${ }^{a}$ Bioproducts Discovery and Development Centre, Department of Plant Agriculture, University of Guelph, Crop Science Building, 50 Stone Road East, Guelph, ON, N1G 2W1, Canada.E-mail: mohanty@uoguelph.ca

${ }^{b}$ School of Engineering, University of Guelph, Thornbrough Building, 50 Stone Road East, Guelph, ON, N1G 2W1, Canada

$\dagger$ Electronic supplementary information (ESI) available. See DOI: 10.1039/c8ra01707g
}

have existed in the market for many years, such as Orgalloy® resins from Arkema, with applications in the automotive sector and in cable sheathing. ${ }^{1}$

Due to the inherent immiscibility between nylon and PP, there is difficulty in achieving a blend with good distribution of the dispersed phase and desirable properties. Two main mechanisms control the blend morphology: particle breakup and coalescence, as described elsewhere. ${ }^{2}$ It is widely accepted that particle breakup is influenced by two competitive forces: the flow stress tending to deform the particle, and the interfacial stress tending to minimize the interfacial area. This breakup mechanism is in competition with droplet coalescence. Coalescence occurs via the droplet collisions induced by differences in velocity. The interaction increase induced by the proximity of particles in the molten polymer results in their deformation, followed by their coalescence. The mechanisms of coalescence and breakup during melt mixing are controlled by multiple parameters such as the blend composition, interfacial tension, shear stress, molecular weight, and viscosity ratio. 
The blend composition is the dominant parameter - when a higher concentration of the dispersed phase is added, more particles undergo particle-particle collisions and consequently an increase in the size of coalescing particles is observed. A lower interfacial tension would reduce the particle size of the dispersed phase by increasing the interfacial adhesion between the phases. To achieve this, the use of compatibilizer is frequently employed. Though the mechanism occurring during the blending of different components in a barrel is not fully understood, three main mechanisms are proposed. The compatibilizer would allow for a decrease in interfacial tension between the phases, facilitating the formation and stability of co-continuous structures. The second mechanism assumes that the compatibilizer would suppress the inter-droplet flow circulation, resulting in a decrease in mobility of the interface, also called Marangoni effect. The last theory proposes that surface active droplets containing the block copolymer would be affected by steric repulsive forces when in the vicinity of each other, preventing the mechanism of coalescence from occurring. Therefore, it is generally accepted that the compatibilizer mainly acts on the mechanism of droplet coalescence, rather than the breakup mechanism, by suppressing coalescence. On the other hand, it was observed by Park et al. that a lower molecular weight is beneficial to droplet breakup. ${ }^{3}$ However, in the presence of a compatibilizer, the effect of molecular weight would be suppressed by the presence of the active copolymer at the particles' interface. In addition, it is generally observed that the dispersed phase would be finely dispersed if its viscosity is lower than that of the matrix. The viscosity ratio is commonly used, and a value close to unity would result in a smaller droplet size. Different theories were proposed to predict the mechanisms of droplet breakup. One of the most recognized is the Taylor's theory stating that the droplet breakup would be enhanced by high matrix viscosity, high shear rate, large droplet size and a small interfacial tension.

In order to produce blends with good mechanical properties, compatibilization, is an essential step. The addition of a compatibilizing agent allows a better dispersion and interfacial adhesion between the two phases. A well-studied compatibilization route for these polymers is through melt blending with maleic anhydride grafted polypropylene (PP- $g$ MA). ${ }^{4,5}$ This approach has been studied by many researchers, with a general consensus that PP-g-MA efficiently reduces the tension between PA6 and PP. ${ }^{4,6-11}$ Reduced interfacial tension after reactive extrusion with PP- $g$-MA has been consistently confirmed through reduction of droplet size as observed via microscopic analyses. ${ }^{4,6,9,10}$ While the improvement of mechanical properties with PP-g-MA addition is evident, PA6/ PP blends are outperformed in terms of strength and stiffness by pure PA6 as a natural consequence of the comparatively low strength and modulus of PP. Even in well-compatibilized blends of PA6/PP, strength and modulus decrease proportionally with increasing PP content. In this study, a solution to this shortcoming was proposed via the inclusion of a third polymer, poly(lactic acid) (PLA), to form ternary polymer blends of PA6/PP/PLA. PLA is a renewable, bio-based polyester with excellent tensile and flexural strength and modulus surpassing that of PA6. Therefore, the addition of low molecular weight PLA would increase the biobased content of the material while helping the injection molding in the molten state.

The use of blends in industry is a common and low cost practice to combine into a single material the properties of its individual components. In the literature, the addition of premade block or graft copolymers, or the reactive compatibilization in situ, are strategies commonly used in binary blend compatibilization. Due to the difficulty of achieving phase compatibilization, ternary blends have received less attention. ${ }^{12-16}$ Most of the literature on ternary blends refers to a matrix (usually polar) and two dispersed phases (usually apolar) compatibilized by two compatibilizers or multi-phase compatibilizers. Though from a morphological perspective the polymer distribution is generally improved by compatibilization, the mechanical performance is not always significantly improved compared to the uncompatibilized blends. The synthesis of bi-functional compatibilizers containing the chemical groups of the respective polymer phases has been applied successfully, and the use of a tough dispersed phase showed significantly improved strain at break. ${ }^{17,18}$

Mainly influenced by the viscosity of the individual polymers, their polarity, surface tension, and miscibility, a prediction of the morphology of binary blend systems can be made. Blending three or more polymers gives rise to more complex interactions in which the composition and processing parameters play a significant role in the material morphology. The influence of multi-phase morphologies on rheological behaviour and mechanical properties has fostered a special interest from the scientific community in the development of models predicting blend morphologies based on viscosity measurements and interfacial tensions. Hobbs et al. and later Luzinov et al., Guo et al., etc. proposed a model predicting the morphology of ternary blends..$^{14,15,19-23}$ In addition, the thermal treatment (temperature and time) can also influence the morphology of the polymer blends. ${ }^{24,25}$ In a ternary blend, containing one main phase and two dispersed phases, four different morphologies can be expected: the encapsulation of one dispersed component by the other resulting in a core-shell structure (two morphologies possible depending on the encapsulation of the dispersed phase 1 on 2 or 2 on 1), the distinct separation of multiphase droplets, or finally an intermediate state where no organization prevails.

In this work, the process was simplified by using a commercial compatibilizer and selecting appropriate polymers. Our strategy here was to combine both a chemical and a physical effect to achieve fine dispersions of sufficient affinity. In one way, this target was achieved by using a maleated compatibilizer, PP- $g$-MA, while the selection of polymers of appropriate molecular weights assured a good dispersion while maintaining mechanical performance. The mechanical properties are presented in a concurrent manuscript. ${ }^{26}$ 


\section{Experimental}

\subsection{Materials}

The polyamide 6 used in this work was Ultramid B27E, a lowviscosity extrusion grade polymer supplied by BASF (Germany), referred to as PA6. PA6 is characterized by a density of $1.12 \mathrm{~g} \mathrm{~cm}^{-3}$, a melting point of $220^{\circ} \mathrm{C}$ and the weight average molecular weight $\left(M_{\mathrm{w}}\right)$ of B27E is $65200 \mathrm{~g} \mathrm{~mol}^{-1} \cdot{ }^{27}$ Ingeo Biopolymer 3251D, an injection-grade PLA from NatureWorks (USA), was used as the PLA phase. PLA is characterized by a density of $1.25 \mathrm{~g} \mathrm{~cm}^{-3}$, a melting point of $170{ }^{\circ} \mathrm{C}$ and the weight average molecular weight $\left(M_{\mathrm{w}}\right)$ of $3251 \mathrm{D}$ is $55400 \mathrm{~g}$ $\mathrm{mol}^{-1} \cdot{ }^{28}$ PP $1120 \mathrm{H}$ supplied by Pinnacle Polymers (USA) was used as the PP phase. PP is characterized by a density of $0.9 \mathrm{~g} \mathrm{~cm}^{-3}$, a melting point of $164{ }^{\circ} \mathrm{C}$ and the weight average molecular weight $\left(M_{\mathrm{w}}\right)$ of $\mathrm{PP} 1120 \mathrm{H}$ is $170000 \mathrm{~g} \mathrm{~mol}^{-1} \cdot{ }^{29}$ Fusabond P353 from DuPont (USA) was selected as the maleated polypropylene compatibilizing agent. The maleation grade of this compatibilizer has been determined to be in the range of 1.4 to $1.9 \% .^{4,30}$

\subsection{Blend preparation}

PA6 and PLA were dried overnight at $80{ }^{\circ} \mathrm{C}$ to eliminate moisture content which can lead to hydrolysis. Blends were prepared via melt processing in a Leistritz Micro-27 (Germany) twin-screw extruder in co-rotation configuration operated at 100 RPM (residence time $\sim 90$ seconds). All components were pre-mixed and fed from a single feeder at a feed rate of $7 \mathrm{~kg}_{\text {hour }}{ }^{-1}$. The configuration of the 12 heating zones set at $\sim 250{ }^{\circ} \mathrm{C}$ are shown in Table $\mathrm{S} 1$ in the ESI figures. $\uparrow$ This heating profile was selected to promote reactive extrusion at high temperature in the screw mixing zones, while limiting the time spent at high temperature to reduce degradation of the PLA phase. The reduced temperature at the die also limited expansion of the extrudate and improved the processability of the material.

All blends designed in this study are detailed in Table 1 with the following naming convention: a prefix of B for "binary", T for "ternary", $\mathrm{C}$ for "compatibilized", with the number after the dash corresponding to the weight fraction of PA6 in the blend. For example, BC-80 is a compatibilized blend of PA6 and PP, with a PA6/PP ratio of $80 / 20$. Blends were selected to investigate the properties of compatibilized and uncompatibilized PA6/ PLA/PP ternary blends in parallel with equivalent PA6/PP blends. In the first section, a full blending profile of binary blends of PA6/PP was developed to analyze the role of the compatibilizer in the binary blend. In the second section, ternary blends of PA6/PLA/PP were produced following parallel blending ratios to the binary blends. In all compatibilized blends, $5 \%$ PP- $g$-MA by weight was added, sacrificing an equivalent amount of the PP fraction. A constant 5\% PP-g-MA was selected after preliminary investigations on formulations processed with 2.5, 5 and $7.5 \mathrm{wt} \%$ (see Fig. S1 in ESI $\dagger$ ). SEM imaging of ternary blends showed a significant PP particle size reduction after addition of $2.5 \% \mathrm{PP}-g$-MA, while an increase of the mechanical properties was noticed. These behaviors were enhanced after raising the concentration up to $5 \%$ PP- $g$-MA, in
Table 1 Blending ratios for ternary and binary blends of PA6, PLA and PP

\begin{tabular}{|c|c|c|c|c|c|c|}
\hline & & \multirow[b]{2}{*}{ Name } & \multicolumn{4}{|c|}{ Component (wt\%) } \\
\hline & & & PA6 & PLA & PP & PP- $g$-MA \\
\hline & Neat polymers & Neat PA6 & 100 & 0 & 0 & 0 \\
\hline & & Neat PLA & 0 & 100 & 0 & 0 \\
\hline & & Neat PP & 0 & 0 & 100 & 0 \\
\hline \multirow[t]{18}{*}{ Binary } & Uncompatibilized & B-90 & 90 & 0 & 10 & 0 \\
\hline & & B-80 & 80 & 0 & 20 & 0 \\
\hline & & B-70 & 70 & 0 & 30 & 0 \\
\hline & & B-60 & 60 & 0 & 40 & 0 \\
\hline & & B-50 & 50 & 0 & 50 & 0 \\
\hline & & B-40 & 40 & 0 & 60 & 0 \\
\hline & & B-30 & 30 & 0 & 70 & 0 \\
\hline & & B-20 & 20 & 0 & 80 & 0 \\
\hline & & B-10 & 10 & 0 & 90 & 0 \\
\hline & Compatibilized & BC-90 & 90 & 0 & 5 & 5 \\
\hline & & BC-80 & 80 & 0 & 15 & 5 \\
\hline & & BC-70 & 70 & 0 & 25 & 5 \\
\hline & & BC-60 & 60 & 0 & 35 & 5 \\
\hline & & BC-50 & 50 & 0 & 45 & 5 \\
\hline & & BC-40 & 40 & 0 & 55 & 5 \\
\hline & & BC-30 & 30 & 0 & 65 & 5 \\
\hline & & BC-20 & 20 & 0 & 75 & 5 \\
\hline & & BC-10 & 10 & 0 & 85 & 5 \\
\hline \multirow[t]{8}{*}{ Ternary } & Uncompatibilized & $\mathrm{T}-90$ & 90 & 5 & 5 & 0 \\
\hline & & T-80 & 80 & 10 & 10 & 0 \\
\hline & & $\mathrm{T}-70$ & 70 & 15 & 15 & 0 \\
\hline & & $\mathrm{T}-60$ & 60 & 20 & 20 & 0 \\
\hline & Compatibilized & TC-90 & 90 & 5 & 0 & 5 \\
\hline & & TC-80 & 80 & 10 & 5 & 5 \\
\hline & & TC-70 & 70 & 15 & 10 & 5 \\
\hline & & TC-60 & 60 & 20 & 15 & 5 \\
\hline
\end{tabular}

particular the elongation at break. The further addition of PP- $g$ MA to $7.5 \%$ did not show any further particle size reduction, but slightly decreased the mechanical properties. Due to the reduced melt strength of the uncompatibilized ternary blends, the ternary blends were only investigated to a blending ratio of 60/20/20 PA6/PLA/PP. Homologous blends were formulated to contain equivalent weight fractions of PA6. Each of the neat polymers was also extruded and injection moulded in the same fashion to give baseline data for comparison.

\subsection{Morphological analysis}

The morphology of the blends was investigated using both scanning electron microscopy (SEM) and atomic force microscopy (AFM). The SEM used in the work was a Phenom ProX (Netherlands) set to an accelerating voltage of $10 \mathrm{kV}$. All specimens tested in the SEM were impact fracture surfaces from the Izod impact tests of the blends. Certain specimens were etched in chloroform for 24 hours to remove the PLA phase. Droplet analysis of SEM micrographs was performed using ImageJ software.

The AFM used in the study was a Bruker (USA) Nanoscope Multimode 8 in peak force quantitative nano-mechanical (PFQNM) mode. Samples were prepared for the AFM using 
a Leica (Germany) EM UC7 ultramicrotome with a diamond knife to produce smooth, flat regions for AFM scanning.

\subsection{Rheological analysis}

The rheology measurements were performed using a MCR302 rheometer (Anton Paar $\mathrm{GmbH}$ ) using a parallel plate configuration in oscillatory mode. The plate diameter used was $25 \mathrm{~mm}$, with a measurement gap distance set at $1 \mathrm{~mm}$. A strain of $10 \%$ was applied which was determined to be within the linear viscoelastic region. Sweeps in the range of $0.1-600 \mathrm{rad} \mathrm{s}^{-1}$ were performed from high to low frequencies. The rheological behaviour of neat PA6, neat PP and their binary and ternary blends were conducted at the processing temperatures $\left(250^{\circ} \mathrm{C}\right)$ under nitrogen atmosphere. At the temperature investigated, the viscosity of PLA was too low to be recorded.

\subsection{Contact angle measurements}

The contact angle measurements of the extruded resins were done at room temperature on a goniometer/tensiometer Model 260 (Ramé-Hart Instruments Co) employing water, ethylene glycol and diiodomethane as liquids. The static sessile drop method was applied at 50\% humidity on injection-moulded samples. The contact angle was measured six times, on two different regions of two surfaces, per sample.

\section{Results and discussion}

\subsection{Blend morphology}

3.1.1 SEM analysis. Scanning electron microscopy (SEM) was used to analyze the comparative morphology and distribution of each polymer phase in the ternary and binary blends. The blending ratios and naming conventions used are elaborated in the experimental section. In the B-80, there are spherical domains of PP dispersed in the continuous PA6 phase in a sea-island structure, with diameters ranging from 2-10 microns (Fig. 1a). The poor miscibility of PP with PA6 is wellstudied, and similar dispersion of PP domains in PA6 have been observed in many studies. ${ }^{4,7,11}$ As the PP weight fraction was increased (Fig. 1b-d), the size of the dispersed domains increased. As introduced earlier, when a higher concentration of PP is added, more PP particles undergo particle-particle collisions and consequently an increase in the size of coalescing particles is observed. The aspect ratio of the dispersed domains also increased with increases in the PP fraction, as the larger domains were deformed by shearing force during blending and injection. As the PA6/PP blending ratio reached 60/40 in B-60 (Fig. 1b), a co-continuous morphology was developed. Continuous domains of PP are distributed and interwoven among the continuous PA6 phase, with small droplets of PP also dispersed in the PA6 phase. This co-continuous behaviour was also observed in B-50, after which phase inversion occurred. Blends B-40, B-30, B-20 and B-10 all showed dispersed domains of PA6 within a continuous PP matrix, with the domain size reducing with decreasing PA6 concentration; B-40 and B-20 are shown in Fig. 1c and d, respectively. In all binary blends, the interaction between the two phases was very limited, as is evident from the

\section{$40 \mu \mathrm{m}$}
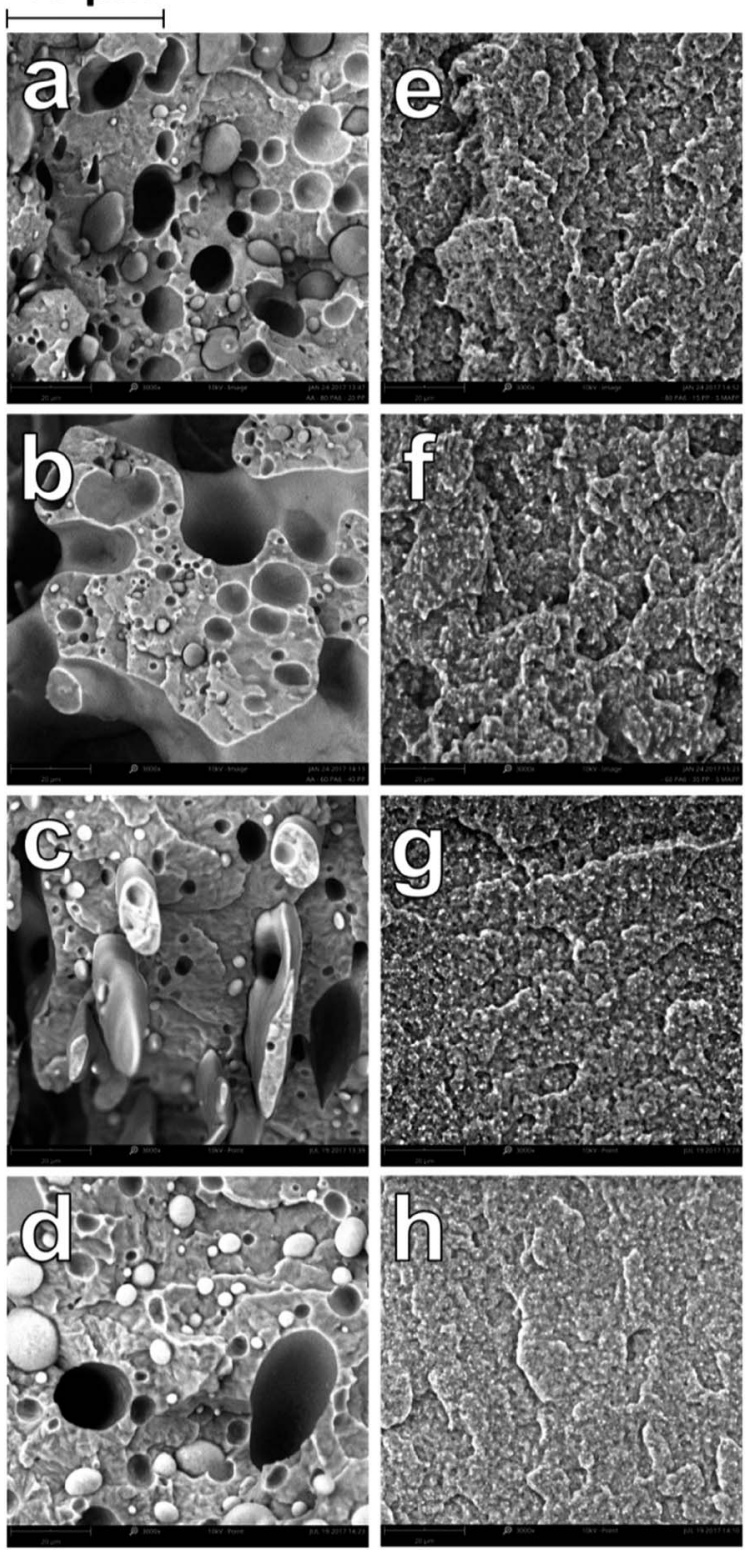

Fig. 1 SEM micrographs at $3000 \times$ magnification of impact fracture surfaces of binary blends (a) B-80 (b) B-60 (c) B-40 (d) B-20 (e) BC-80 (f) BC-60 (g) BC-40 (h) BC-20.

visible gaps at the interface of the phases, and the clean pullout of the dispersed domains. Large voids are visible due to the pullout of PP domains from the PA6 matrix during impact fracture due to the poor adhesion of the polymers. SEM study of these binary systems very clearly illustrates the poor compatibility between PA6 and PP.

A substantial change in the morphology of the binary blends was observed with the addition of PP- $g$-MA as a compatibilizer. As seen in Fig. 1e-h, at all blending ratios, the addition of PP-gMA dramatically improves the miscibility of the PP and the PA6, resulting in an incredibly stark contrast in morphology compared to the homologous non-compatibilized blends in Fig. 1a-d. At a magnification of $12000 \times$ in SEM imaging, the dispersed phase in the compatibilized blends becomes evident. 


\section{$5 \mu \mathrm{m}$}
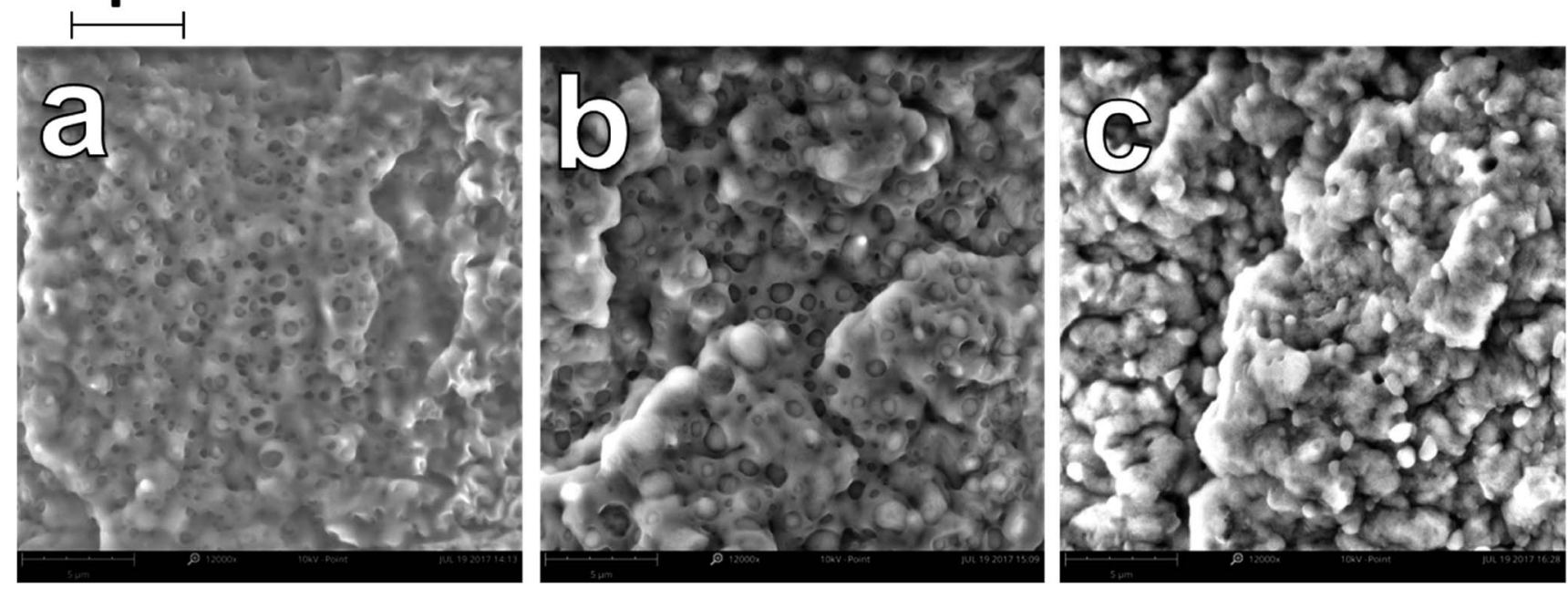

Fig. 2 SEM micrographs at $12000 \times$ magnification of impact fracture surfaces of (a) BC-20 (b) BC-50 (c) BC-80

The size of the dispersed domains is reduced to below 1 micron, and adhesion between the dispersed phase and the continuous phase is unambiguous in Fig. 2.

Furthermore, the dispersed phases were very well distributed and nearly uniform in size, in stark contrast to the large irregular domains observed in the non-compatibilized blends. Due to this enhanced adhesion between the two phases, pullout of the dispersed phases is dramatically reduced. On a macro scale, impact fractured surfaces of compatibilized blends were nearly indistinguishable from those of the neat polymers. This change in morphology is typical in PA6/PP blends compatibilized with PP- $g$-MA. ${ }^{4,6,11,31}$ Reactive extrusion at high temperature promotes a reaction between PP- $g$-MA and PA6, via a two-step reaction between the diamine terminal groups of the PA6 and the anhydride groups in the PP-g-MA. ${ }^{7,32-34}$ This yields a graft copolymer of PP-g-PA6 linked via an imide bond; the reaction mechanism is depicted in Fig. 3. The grafting of maleic anhydride grafted polypropylene onto nylon 6 was studied by ATRFTIR mapping, showing an increase of the miscibility of the polymers after the addition of compatibilizer. ${ }^{35}$ The authors also found that $6 \mathrm{wt} \%$ of compatibilizer yielded the optimal miscibility. This graft copolymer can interact with both the PA6 and PP phases, creating an interface between the phases and improving their adhesion and miscibility. ${ }^{4,6,734}$ The reason is that, in uncompatibilized blends, molecules of PP at the interface with PA6 have less interaction (hydrogen bonding, van der Waals forces, polar interactions, etc.) with PA6 molecules than with other PP molecules. The addition of a compatibilizer composed of "reactive" molecules of PP lowers the interaction force between PP and PP-g-MA on one side of the interface, and on the other side reacts with PA6, which results in a further increase of the cohesive energy i.e. decrease of interfacial tension.

The uncompatibilized ternary blends also demonstrated a dispersion of spherical domains of immiscible polymers within a continuous PA6 matrix. Fig. 4a-d shows the evolution of the dispersed phase structures from T-90 to T-60. There are evident gaps between the dispersed phases and the PA6 due to the immiscibility of the polymers, as well as large voids from pullout during impact fracture. In order to identify the dispersed phases, additional samples were prepared by etching the PLA phase with chloroform. Etching of PLA provided a clear understanding of the how the two phases were dispersed, as seen in Fig. 5. In T-90 (Fig. 5a), PP domains with a diameter between 2-3 microns are present, as well as voids left from the pullout of these domains. Small voids from the etched PLA phase were present on the scale of $0.2-0.8 \mu \mathrm{m}$, and very well dispersed through the PA6 main phase. Similarly in T-70 (Fig. 5c), large PP voids and pullouts were observed between 5-20 $\mu \mathrm{m}$ in diameter, while PLA was dispersed in 0.4-1.4 $\mu \mathrm{m}$ droplets.

Interaction between the two dispersed phases of PLA and PP is minimal due to the poor miscibility of these polymers. ${ }^{36}$ In contrast to the binary blend, it is evident upon inspection that there are two distinct dispersed species. The two polymers formed separate, dispersed domains, which had a smaller average diameter than the PP domains in the binary blend due to the lower relative concentration of each polymer compared to the major PA6 phase. As the weight fraction of PP and PLA increased, the size of the dispersed domains also increased. Etching in chloroform clearly showed that the PLA droplets were much smaller than those of PP, for the same concentration. In all ternary blends, the PLA was very finely dispersed; PP and PLA are also poorly miscible with each other and thus disperse separately, preventing the formation of co-continuous structures at the tested blending ratios. ${ }^{36}$ As observed in the binary blend, as the PP domains increased in size, they were more affected by shearing forces and became increasingly irregular in size and shape.

With the addition of PP-g-MA, a similar compatibilizing effect was observed in the ternary blends. As seen in Fig. 4e-h, the morphology of the compatibilized ternary blends was vastly 


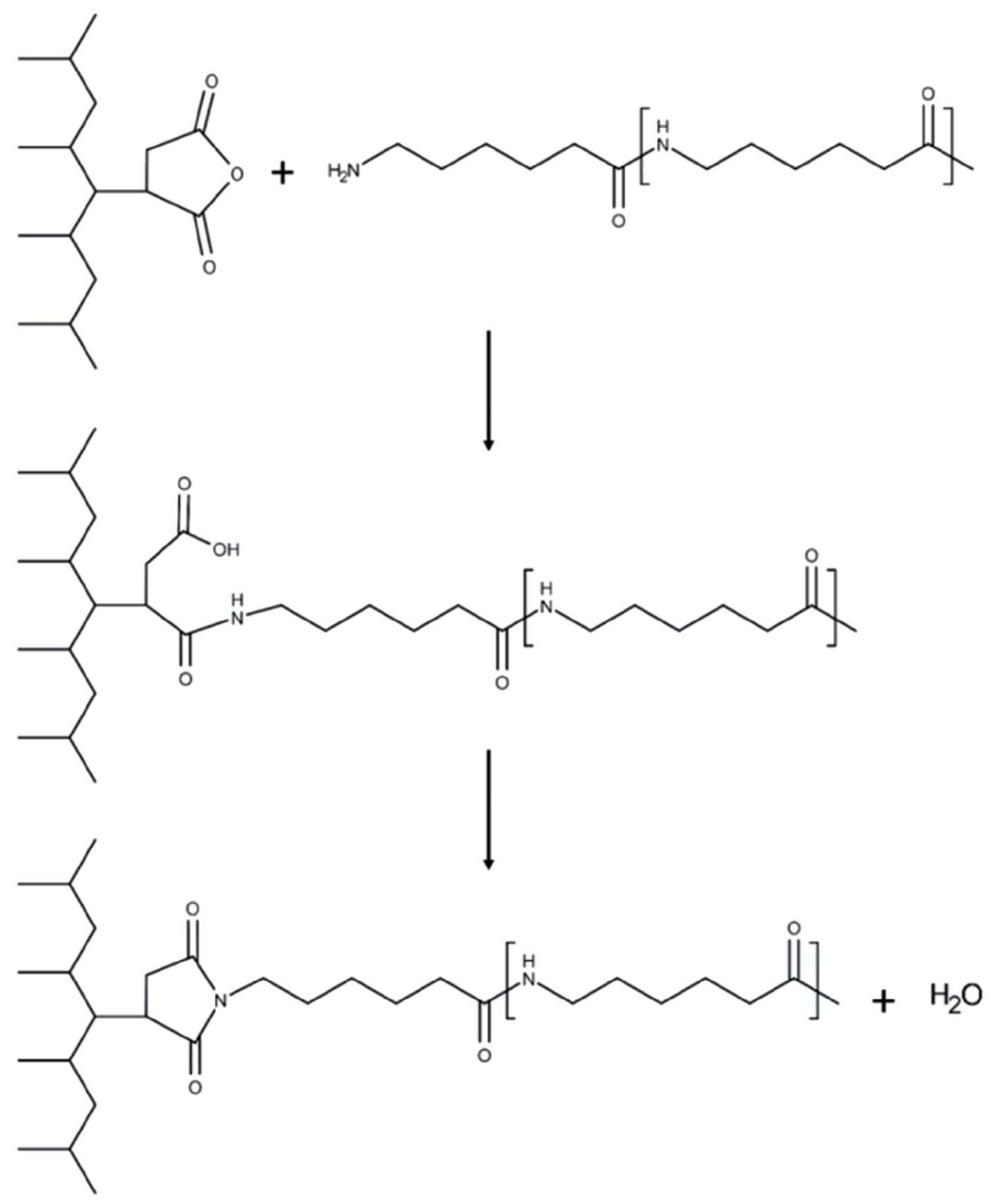

Fig. 3 Grafting mechanism of PP-g-MA and PA6 during reactive extrusion.

different due to the improved miscibility and adhesion between the PA6 and PP phases. Closer inspection of etched samples of TC-90 (Fig. 5b) and TC-70 (Fig. 5d) show that as in the binary blends, the compatibility of PP with PA6 was dramatically improved. Dispersed PP domains were revealed at high magnification in Fig. 6, similar to the droplet size observed in the binary blends (Fig. 2). The presence of PLA in the system does not interfere with the grafting between PA6 and PP- $g$-MA in the ternary blend. Etching of PLA in Fig. 5 showed that PP- $g$-MA addition had no clear impact on the droplet size of PLA. Image analysis revealed that PLA droplets in T-90 and T-70 had average diameters of 0.41 and $0.52 \mu \mathrm{m}$, respectively; in TC-90 and TC-70 these values were 0.40 and $0.53 \mu \mathrm{m}$ respectively.

PP- $g$-MA has been demonstrated to reduce tension in binary PP/PLA systems by both Yoo et al. and Choudhary et al. ${ }^{36,37}$ While grafting between the maleic anhydride groups of PP- $g$-MA and carboxylic acid terminal groups of PLA is less favourable than PP- $g$-PA6 grafting due to the relatively high nucleophilicity of the diamine groups, it is possible that some grafting occurred between PLA and PP- $g$-MA, reducing the interfacial tension between the PLA and PP phases. ${ }^{36}$ Interestingly, in TC-70 (Fig. 6), the PP droplets were clustered at the perimeter of etched PLA droplets, a phenomenon that was only observed in the compatibilized blend. It is possible that due to an improved affinity between PP and PLA, droplets of PP in the compatibilized system were localized to the PA6/PLA boundary.

3.1.2 AFM analysis. Atomic force microscopy (AFM) was performed in parallel with the SEM study to provide additional information and further investigate the effect of PP-g-MA on the dispersion of the PLA and PP phases. AFM was used to scan highly smoothed sample surfaces prepared with a microtome, allowing the distinction of the separate phases based on their stiffness, measured as Derjaguin-Muller-Toporov (DMT) modulus.

Fig. 7 compares the distribution of polymer phases on a 20micron scale in binary and ternary blends containing $80 \%$ PA6, 

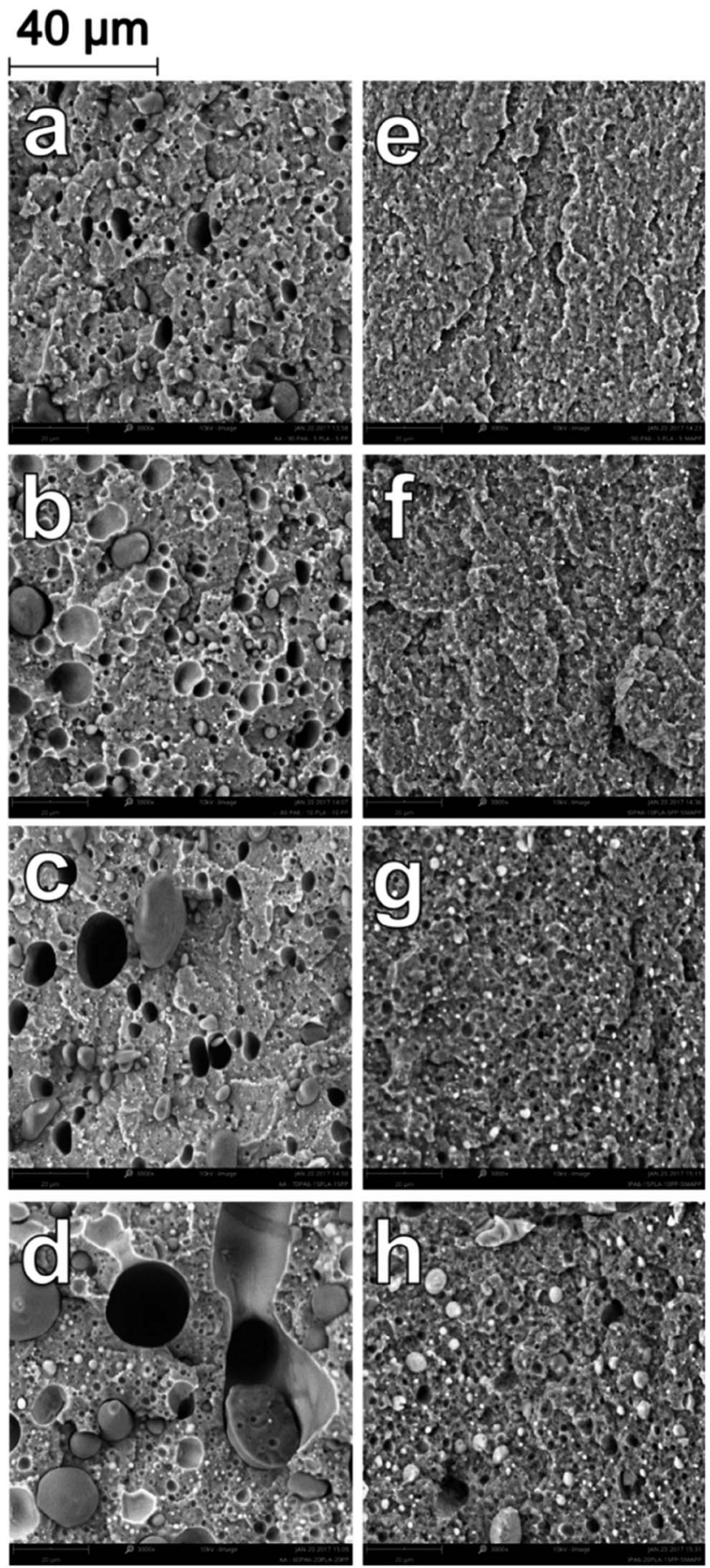

Fig. 4 SEM micrographs at $3000 \times$ magnification of impact fracture surfaces of ternary blends (a) T-90 (b) T-80 (c) T-70 (d) T-60 (e) TC-90 (f) TC-80 (g) TC-70 (h) TC-60.

with and without the addition of PP- $g$-MA as compatibilizer. In T-80 (Fig. 7c), there are multiple distinct peaks of stiffness, which indicate dispersed domains of PLA. PLA domains stands out as sharp peaks in DMT modulus, due to the high stiffness of PLA (3.3 GPa) relative to the PA6 $(2.7 \mathrm{GPa})$ and PP (1.9 GPa) phases. With the addition of PP- $g$-MA compatibilizer in TC-80 (Fig. 7d), a remarkable change in phase morphology was observed. The PLA and PP phases are clearly well distributed throughout the PA6 phase, as demonstrated by the distribution of DMT modulus peaks in Fig. 7b. The modulus peaks attributed to PLA are very well dispersed throughout the polymer matrix, as are the darker regions corresponding to PP. This result correlates strongly with the observations in the SEM, demonstrating that the addition of PP-g-MA resulted in improved compatibility of the PP phase and that both PP and PLA are well dispersed in the compatibilized blends.

A similar distinction in morphology was evident between the binary blends with the addition of PP- $g$-MA. In the noncompatibilized blend B-80 (Fig. 7a), large PP domains ranging from 5-10 $\mu \mathrm{m}$ can be distinguished within the continuous PA6 phase. Topographical data shows not only the size of these domains, but gaps between the PP domains and the PA6 phase due to the relative immiscibility of the polymers. This supports the observation of similar voids in the SEM analysis, which allowed easy pullout of the PP domains during impact fracture of the material. In both the binary blends and ternary blends, AFM scanning revealed that the addition of PP- $g$-MA results in a much more homogenous modulus distribution, which is evidence of the improved compatibility and dispersion of the PP and PLA phases within the PA6 phase. ${ }^{4}$

\subsection{Surface tension investigation}

The measurement of the surface and interfacial tension between different polymers of a blend is of significant importance for predicting the wettability, interaction and morphology of the blend components. Because this combination of polymers has not been previously studied in the literature, and to limit the uncertainty linked to multiple source of datas, the contact angles were measured via the sessile drop method, and the Owens-Wendt theory (geometric method) was applied to calculate the surface and interfacial tensions of PA6, PLA, PP and their blends. ${ }^{38}$

Focusing on the homopolymers, both the surface tension and associated components are significantly influenced by the polymer structure, as shown in Table 2. PA6 shows a higher polar component $\left(\sim 9 \mathrm{mN} \mathrm{m}^{-1}\right)$ than PLA $\left(\sim 4 \mathrm{mN} \mathrm{m}^{-1}\right)$ and PP $\left(\sim 0.5 \mathrm{mN} \mathrm{m}^{-1}\right)$. The presence of both amine and carboxylic group on the surface of PA6 and carboxylic acid group on PLA increase the polarity of the polymer in comparison to the carbon chain constituting PP. On the other hand, the dispersive component of all polymers is in the same range of values $(\sim 35$

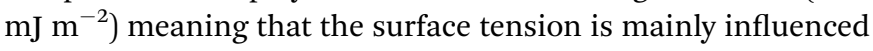
by the polar component i.e. the active groups favouring interactions between the polymers.

The Owens-Wendt theory was applied to calculate the interfacial tension between the pairs of polymers. As expected, the pair PA6/PP shows a high interfacial tension $\left(5.54 \mathrm{~mJ} \mathrm{~m}^{-2}\right)$ highlighting a low affinity between the PA6 and PP phases. The high polarity of PA6 blended with the apolar PP is not favourable from a thermodynamic point of view, which results in clearly separated phases, as seen previously by morphological investigations. Interaction between PLA/PP (1.82 $\left.\mathrm{mJ} \mathrm{m}^{-2}\right)$ appears to be significantly less favourable than between PA6/ 


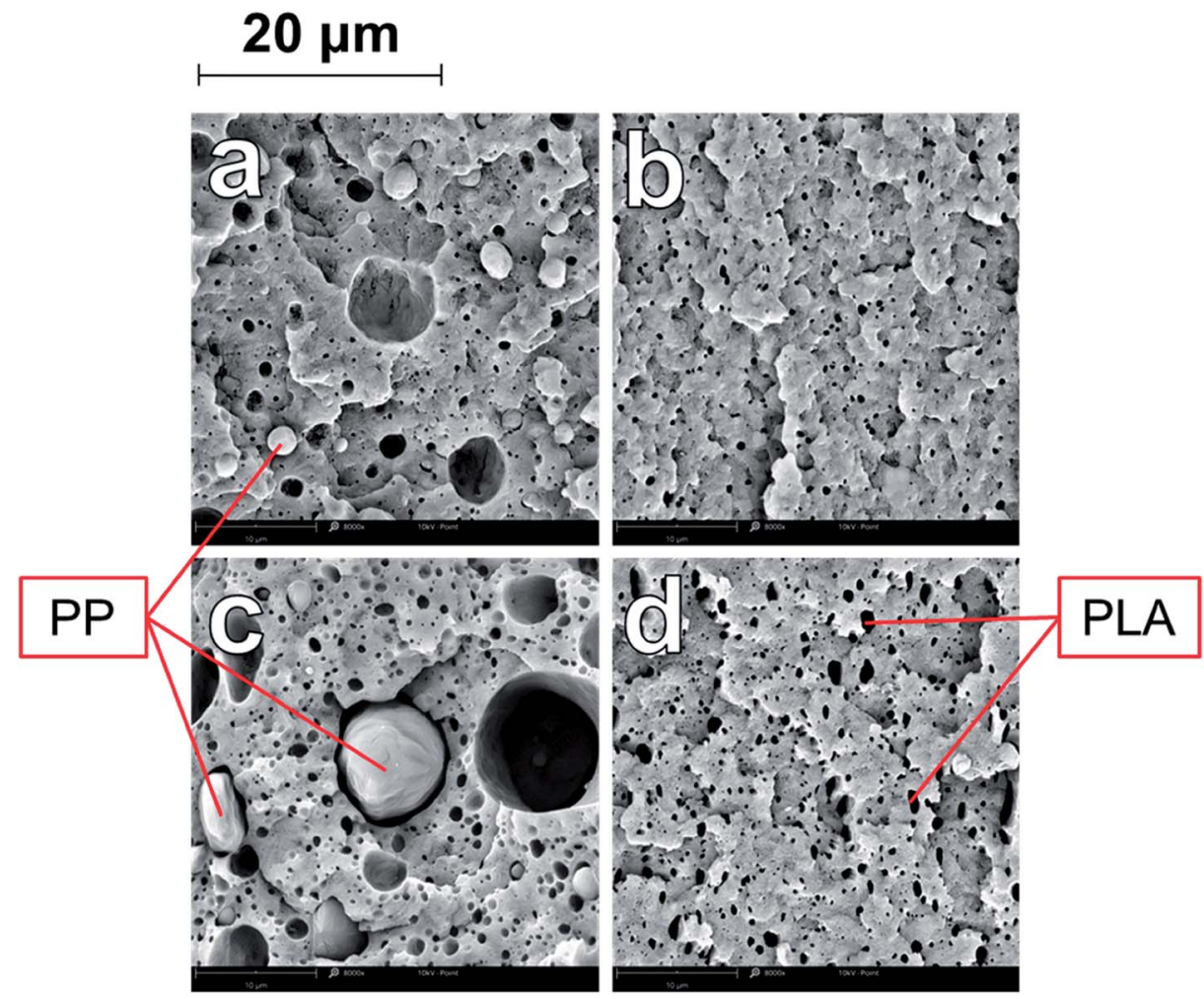

Fig. 5 SEM micrographs at $8000 \times$ magnification of chloroform-etched ternary blends (a) T-90 (b) TC-90 (c) T-70 and (d) TC-70.

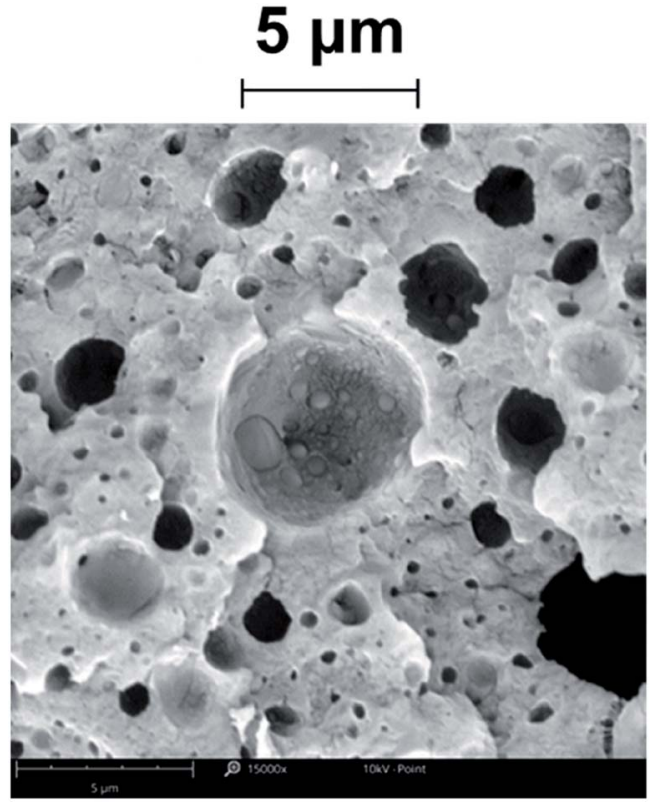

Fig. 6 PP droplets grouped around the perimeter of a chloroformetched PLA droplet, in TC-70 at $15000 \times$ magnification.

PLA (1.06 $\mathrm{mJ} \mathrm{m}^{-2}$ ) which can be explained by the closer tension difference between PA6 and PLA over PLA and PP. The higher the chemical structure affinity, the better the interactions between two phases. The higher affinity between PA6 and PLA support the smaller droplets of PLA $(<1 \mu \mathrm{m})$ observed previously in the uncompatibilized ternary blend (Fig. 4 and 5), in comparison to the PP phase $(<10 \mu \mathrm{m})$. The data measured at room temperature were extrapolated to the processing temperature by application of a temperature coefficient found in the literature: $0.065 \mathrm{~mJ} \mathrm{~m}^{-2}$ for PA6, $0.058 \mathrm{~mJ} \mathrm{~m}^{-2}$ for PP and $0.060 \mathrm{~mJ} \mathrm{~m}^{-2}$ was taken for PLA. ${ }^{39-41}$ At high temperature, the interfacial tension between the different pairs of polymers is reduced, making mixing of the phases easier during the process.

Ternary blends morphology can be further understood and predicted through the application of a theoretical model. While several models have been proposed in the literature, the spreading coefficient model is one of the most known. Hobbs et al. proposed to reuse the Harkin's equation to predict the morphology of immiscible ternary blends. ${ }^{19}$ Thereby, for a ternary system (PA6/PP/PLA) with PA6 as the main phase and PP and PLA the dispersed phases, the spreading coefficient allows one to determine if one of the phases is encapsulated by another. The spreading coefficient $\lambda_{\mathrm{PP} / \mathrm{PLA}}$, aiming to predict if the PP-phase can encapsulate the PLA-phase, can be written as follows:

$$
\lambda_{\mathrm{PP} / \mathrm{PLA}}=\gamma_{\mathrm{PA} 6 / \mathrm{PLA}}-\gamma_{\mathrm{PA} 6 / \mathrm{PP}}-\gamma_{\mathrm{PP} / \mathrm{PLA}}
$$

Similarly, to know if the PLA-phase can encapsulate the PPphase the following formula will be used:

$$
\lambda_{\mathrm{PLA} / \mathrm{PP}}=\gamma_{\mathrm{PA} 6 / \mathrm{PP}}-\gamma_{\mathrm{PA} 6 / \mathrm{PLA}}-\gamma_{\mathrm{PP} / \mathrm{PLA}}
$$



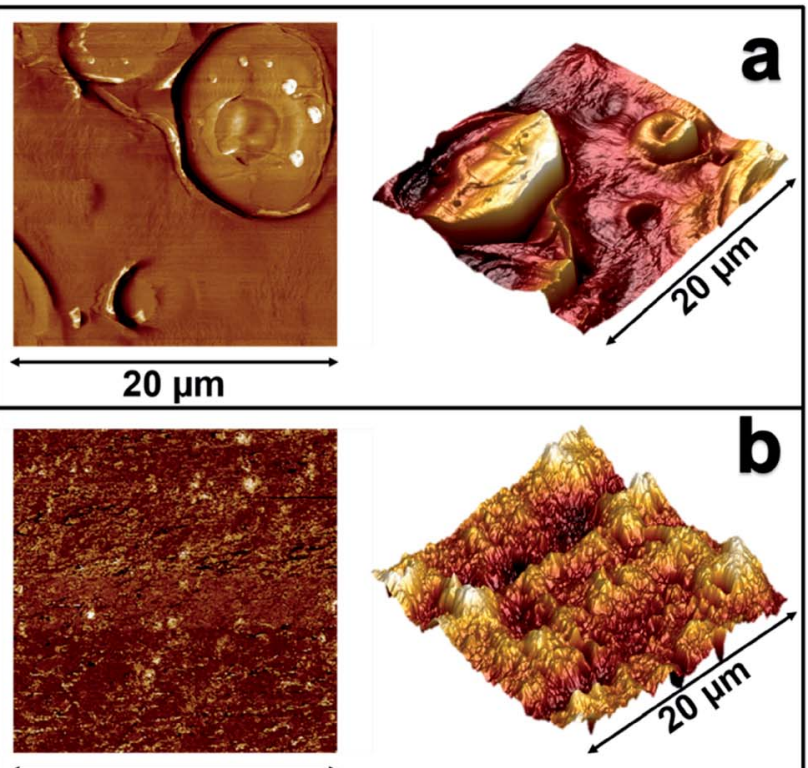

$20 \mu \mathrm{m}$
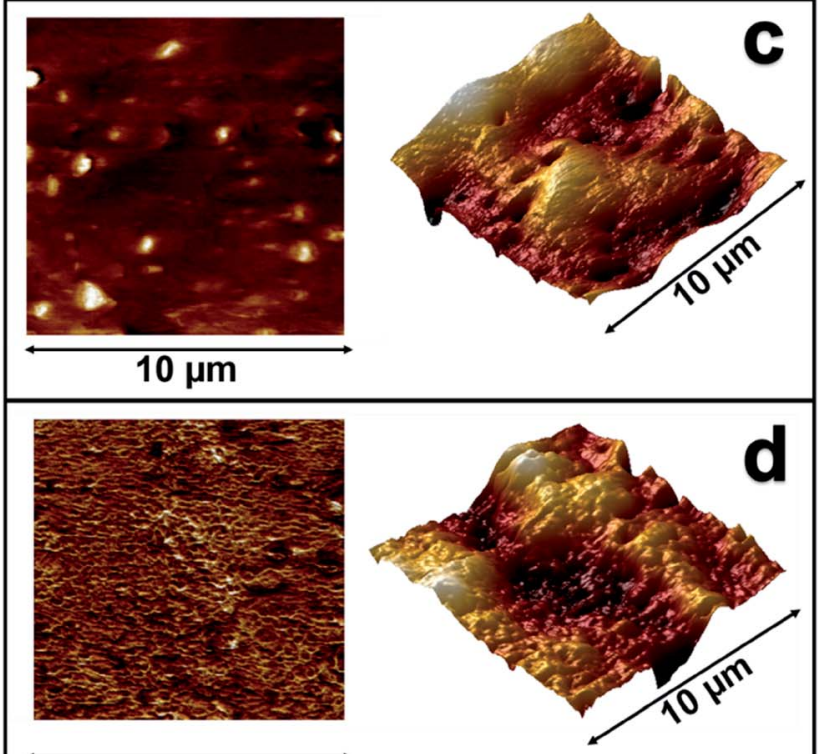

$10 \mu \mathrm{m}$

Fig. 7 AFM scans of blends showing their DMT modulus (left) and 3D topology (right) as measured in $20 \times 20 \mu \mathrm{m}$ scans of (a) B-80, (b) BC80 , and measured in $10 \times 10 \mu \mathrm{m}$ scans of (c) T-80, and (d) TC-80.

With $\lambda_{\mathrm{ij}}$ the spreading coefficient of $\mathrm{i}$ over $\mathrm{j}$, and $\gamma_{\mathrm{ij}}$ the interfacial tension between $i$ and $j$. A positive spreading coefficient value $\lambda_{\mathrm{PP} / \mathrm{PLA}}$ means that the PP-phase is encapsulating the PLA-phase, while a negative value highlights the opposite. If both $\lambda_{\mathrm{PP} / \mathrm{PLA}}$ and $\lambda_{\mathrm{PLA} / \mathrm{PP}}$ are negative, then the PP and PLA phases should remain separate.

The application of this theory shows a negative $\lambda_{\mathrm{PP} / \mathrm{PLA}}$ value (-4.45), meaning that PP-phase would not encapsulate the PLAphase. However, the positive $\lambda_{\text {PLA/PP }}$ value (1.77) indicates that there should be encapsulation of the PP-phase by the PLAphase. From morphological observation, no evidence of encapsulation could be found. The comparison of
Table 2 Surface tension $\gamma_{i}$, interfacial tension $\gamma_{i j}$, and spreading coefficient $\lambda_{i j}$ of PA6, PLA, PP and their blends at both room temperature and extrapolated to the processing temperature

\begin{tabular}{|c|c|c|c|c|c|c|}
\hline \multirow[b]{3}{*}{ Solids } & \multicolumn{6}{|c|}{ Surface free energy parameters $\left(\mathrm{mJ} \mathrm{m}^{-2}\right)$} \\
\hline & $20^{\circ} \mathrm{C}$ & $20^{\circ} \mathrm{C}$ & $20^{\circ} \mathrm{C}$ & $250^{\circ} \mathrm{C}$ & $250^{\circ} \mathrm{C}$ & $250^{\circ} \mathrm{C}$ \\
\hline & $\gamma_{\mathrm{sv}}$ & $\gamma_{S V^{d}}$ & $\gamma_{\mathrm{SV}^{\mathrm{p}}}$ & $\gamma_{\mathrm{sv}}$ & $\gamma_{S^{d}}$ & $\gamma_{\mathrm{SV}^{\mathrm{p}}}$ \\
\hline$\gamma_{\text {PA6 }}$ & 44.7 & 35.3 & 9.4 & 29.8 & 23.4 & 6.4 \\
\hline$\gamma_{\text {PLA }}$ & 41.3 & 37.1 & 4.2 & 27.5 & 24.7 & 2.8 \\
\hline$\gamma_{\mathrm{PP}}$ & 35.3 & 34.8 & 0.5 & 22.0 & 21.6 & 0.4 \\
\hline$\gamma_{\text {PA6/PLA }}$ & 1.06 & & & 0.77 & & \\
\hline$\gamma_{\text {PLA/PP }}$ & 1.82 & & & 1.34 & & \\
\hline$\gamma_{\mathrm{PA6} / \mathrm{PP}}$ & 5.54 & & & 3.88 & & \\
\hline$\lambda_{\mathrm{PLA} / \mathrm{PP}}$ & & & & 1.77 & & \\
\hline$\lambda_{\mathrm{PP} / \mathrm{PLA}}$ & & & & -4.45 & & \\
\hline
\end{tabular}

${ }^{\mathrm{d}}$ Corresponding to dispersive interactions. ${ }^{\mathrm{P}}$ Corresponding to polar interactions.

uncompatibilized ternary blends before and after removing PLA (Fig. 4 and 5) shows a similar gap between the PP droplets and PA6 matrix. Thereby, a second model was applied, this time considering the influence of the interfacial free energy which takes in account both the interfacial tension and the interfacial areas. This minimum free energy model provides a more accurate prediction of the multi-phase morphology. Thence, Guo et al. have proposed an evolution to the model proposed by Hobbs et al. considering both parameters, and successfully predicted the morphologies of ternary and quaternary blends. ${ }^{19,21}$ The equations proposed by Guo et al. were simplified considering that the volume ratio of dispersed phases is the same and assuming that the number of particles of the dispersed phases PP and PLA is identical. This theory is based on the consideration that the morphology of lowest free energy is favourable.

$$
\begin{gathered}
\left(\sum A_{\mathrm{i}} \gamma_{\mathrm{ij}}\right)_{\mathrm{PP}+\mathrm{PLA}}=\left(36 \pi n V^{2}\right)^{1 / 3}\left(\gamma_{\mathrm{PA} / \mathrm{PP}}+\gamma_{\mathrm{PA} / \mathrm{PLA}}\right) \\
\left(\sum A_{\mathrm{i}} \gamma_{\mathrm{ij}}\right)_{\mathrm{PP} / \mathrm{PLA}}=\left(36 \pi n V^{2}\right)^{1 / 3}\left(2^{2 / 3} \gamma_{\mathrm{PA} 6 / \mathrm{PP}}+\gamma_{\mathrm{PP} / \mathrm{PLA}}\right) \\
\left(\sum A_{\mathrm{i}} \gamma_{\mathrm{ij}}\right)_{\mathrm{PLA} / \mathrm{PP}}=\left(36 \pi n V^{2}\right)^{1 / 3}\left(\gamma_{\mathrm{PP} / \mathrm{PLA}}+2^{2 / 3} \gamma_{\mathrm{PA} / \mathrm{PLA}}\right)
\end{gathered}
$$

For those pairs of polymers, the lowest interfacial free energy value was obtained for the PLA/PP structure morphology, meaning that the PLA phase would encapsulate the PP phase. This model is in accordance with the model applied earlier but is not consistent with the morphology observed via SEM. The presence of encapsulation of the PP phase by the PLA could not be distinctly highlighted from morphological observation. The disagreement between theoretical predictions and morphological observation prompted further investigation by rheology to explain the observed morphology.

\subsection{Dynamic rheological behaviour}

To provide more information regarding the interactions of the dispersed phases droplets, frequency sweep tests were 
performed on all samples, both compatibilized and uncompatibilized. The logarithm of the complex viscosity $\left(\eta^{*}\right)$ as a function of the logarithm of sweep frequencies $(\omega)$ was measured at $250{ }^{\circ} \mathrm{C}$ and is shown for PA6, PP and their blends in Fig. 8a and for PA6, PP and PLA and their blends in Fig. 8b. Fig. 8c and $\mathrm{d}$ correspond to the same respective blend compositions to which 5 wt $\%$ of compatibilizer was added. The weight percentage corresponding to each phase is listed in Table 1.

First, we considered the homopolymers, where the flow behaviour mainly depends on the polymer structure and molecular weight. At the temperature investigated, pure PP shows a higher complex viscosity than that of pure PA6 at low shear rate i.e. $<20 \mathrm{rad} \mathrm{s}^{-1}$, which can be explained by the higher molecular weight of PP $\left(M_{\mathrm{w}} \sim 170 \mathrm{~kg} \mathrm{~mol}^{-1}\right)$ over PA6 $\left(M_{\mathrm{w}} \sim 65\right.$ $\left.\mathrm{kg} \mathrm{mol}{ }^{-1}\right)$, (Fig. 8a). The lower molecular weight of PLA $\left(M_{\mathrm{w}} \sim 55\right.$ $\mathrm{kg} \mathrm{mol}^{-1}$ ) in combination with the use of a temperature significantly higher than its melt temperature $\left(+80{ }^{\circ} \mathrm{C}\right)$ prevented the recording of any data. In addition, neat PA6 shows a lower sensitivity to frequencies in comparison to PP, meaning that PA6 has a more Newtonian behaviour than PP, i.e. a limited shear sensitivity. On the other hand, PP is characterized by a short Newtonian region as highlighted by its shear-thinning starting at lower frequencies, beyond $0.4 \mathrm{rad} \mathrm{s}^{-1}$ for PP and $>20 \mathrm{rad} \mathrm{s}^{-1}$ for PA6. In other words, when the shearing increases, the molecular chains slide past each other more easily for PP than PA6, lowering the viscosity. Wu estimated that the molecular weight between entanglements $\left(M_{\mathrm{e}}\right)$ for PA6 is around 2000-2500 $\mathrm{g} \mathrm{mol}^{-1}$, whereas Fetters et al. measured the $M_{\mathrm{e}}$ for PP around 5100-5600 $\mathrm{g} \mathrm{mol}^{-1}$, implying that PA6 has a higher entanglement density than PP. ${ }^{\mathbf{4 2 , 4 3}}$ A higher entanglement density limits the polymer chain flow and consequently the shear-thinning. In this manner, the behaviour observed would possibly be a combination of both a high $M_{\mathrm{w}}$ and a high $M_{\mathrm{e}}$ of PP, resulting in a lower characteristic time for reptation, which is easier to overcome by minor shear strain.

Furthermore, regarding the PLA dispersed phase, its low $M_{\mathrm{w}}$ is associated to a $M_{\mathrm{e}}$ around $4000 \mathrm{~g} \mathrm{~mol}^{-1}$, showing a low density of entanglement. ${ }^{45}$ While the influence of the PLA $M_{\mathrm{w}}$ on the dispersed phase morphology is out of the scope of this work, concurrent studies have already shown the importance of this parameter on the phase distribution, though the conclusions diverge. ${ }^{20,26}$ Some authors have highlighted that the lower the molecular weight of the dispersed phase, the finer the dispersion in the blend. ${ }^{46}$ The considerably low viscosity and $M_{\mathrm{w}}$ of PLA, at such a high temperature, results in an extremely easy flowability, which results in the very small droplets of polymer $(0.4 \mu \mathrm{m})$ observed during the morphological investigation. The disagreement between the morphology theories applied (not taking into account the effect of the viscosity) and the morphology observed by SEM and AFM can be explained by the extremely low viscosity of PLA at the processing temperature (high polymer chain degradation and low density of
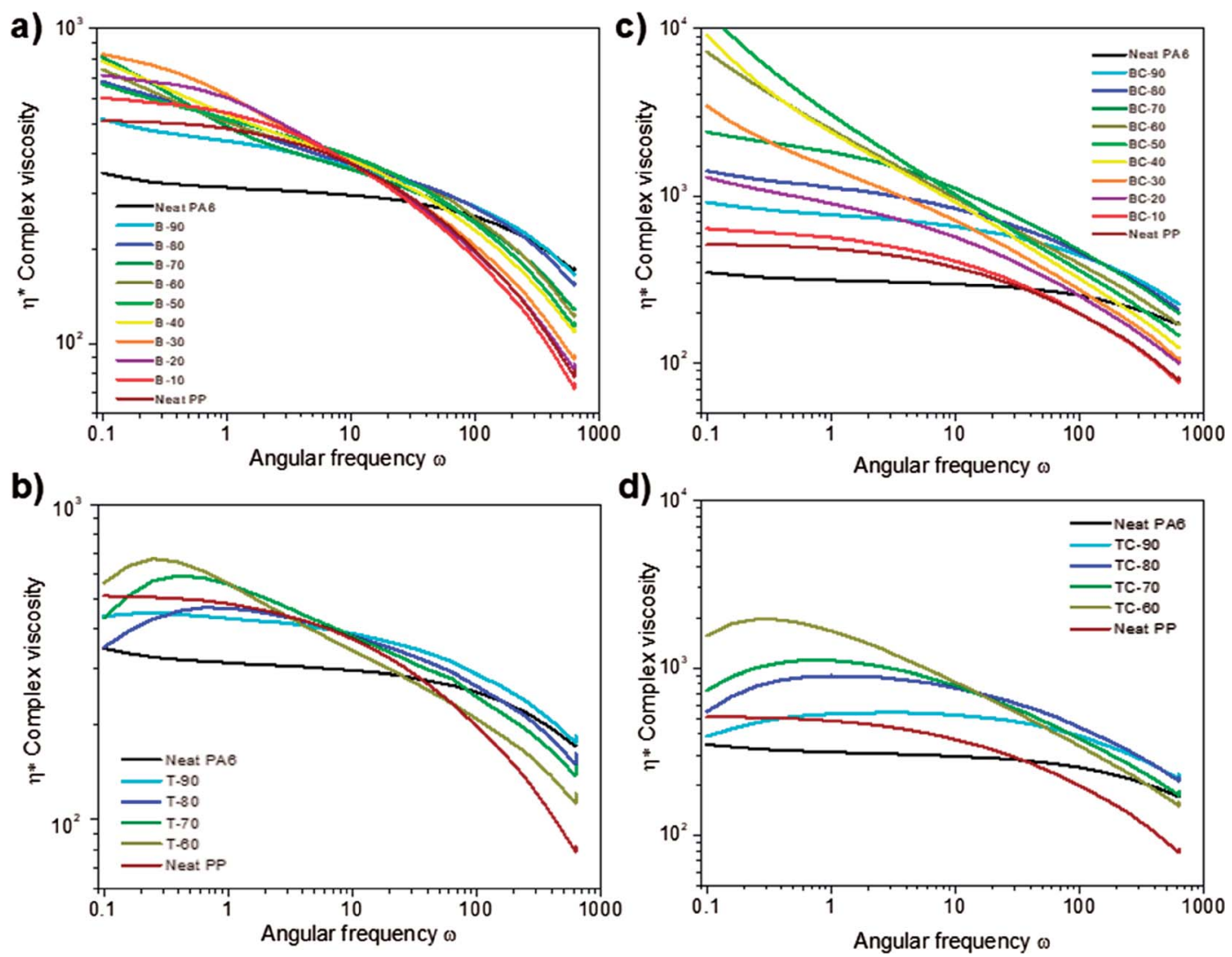

Fig. 8 Complex viscosity versus sweep frequency for (a) uncompatibilized binary blends and (c) compatibilized binary blends at a fixed concentration ( $5 \mathrm{wt} \%$ ) on the full range of ratio, and for (b) uncompatibilized ternary blends and (d) compatibilized ternary blends at a fixed concentration (5 wt\%) on a restricted range of ratio at $250{ }^{\circ} \mathrm{C}$. 
entanglement), making the encapsulation of the PP phase by PLA unfavourable. The effect of the observed morphologies on the mechanical properties is exposed in a concurrent manuscript. ${ }^{26}$

As introduced earlier, the viscosity ratio $(p)$, defined as the ratio of the dispersed phase $\left(\eta_{\mathrm{d}}\right)$ and the matrix $\left(\eta_{\mathrm{m}}\right)$, was calculated for the pairs of polymers PA6/PP and PA6/PLA at 10 $\operatorname{rad~s}^{-1}$. The values measured were lower than 4 , confirming that the particle breakup is made possible as $p_{\mathrm{PA} / \mathrm{PP}}=1.259$ and $p_{\mathrm{PA} / \mathrm{PLA}}=0.108$. Taylor's theory states that a viscosity ratio around unity would be beneficial to the breakup of the minor phase. However, deep studies on the effect of viscosity ratio on blend morphology obtained smaller dispersed phase particle size at lower $p{ }^{44}$ This difference was explained by the effect of the elasticity of the components. According to those results, an easier ability of PLA to flow in comparison to PP would be highlighted by the lower $p$ of PLA.

For blends, the complex viscosity is strongly dependent on many parameters such as composition, miscibility, morphology and the interface, all of which influence the rheological behaviour of the blends. ${ }^{47}$ Focusing on the uncompatibilized binary blends in Fig. 8, all the intermediate ratios show a viscosity higher than that of the neat polymers. This phenomenon is classical for immiscible polymer blends and is characteristic of a higher shear occurring between two immiscible phases. The rheological behaviour of the full range of ratios can be gathered over three main phases: PA6 dominant, PA6 $\sim$ PP equivalent and PP dominant. An overall remark is that the higher the dispersed droplet size, as measured by SEM, the higher the viscosity; the higher friction induced by the particle size is responsible for this change. ${ }^{48}$ In addition, the steady increase of viscosity with decreasing frequency highlights that although the polymers are immiscible when PA6 is the main phase, the chains are easily sliding past each other. An increase of the proportion of PP leads to a shorter Newtonian region with an increasing tendency towards shear-thinning. As expected, the shear-thinning steadily shifts to the lower frequencies with increasing PP content meaning that the extrusion process is made easier by the PP addition i.e. less resistance for the screws and less torque on the motor. The viscoelastic behaviour of the binary blend appears to follow a complex mechanism that can be correlated to the morphology observed earlier by SEM. The relationship between miscibility, phase morphology and rheological behaviour will be further exposed in the Cole-Cole diagram below.

Upon PLA addition, a major difference between the binary and ternary blends is observed as shown in Fig. 8b. A significant complex viscosity drop is observed for all the PLA blends at low frequencies, which is enhanced with increasing PLA concentration. Increasing the PLA content presents a significant advantage for the processing of the blend, as the melt viscosity reduction makes the injection moulding of large and complex moulds easier. This phenomenon is attributed to the depolymerization of the polymer chains over the long duration of the experiment. As already shown in the literature, PLA is sensitive to high temperatures and thus is typically processed at lower temperatures. ${ }^{49}$ Moreover, the addition of PLA gradually increases the non-Newtonian behaviour, shifting the onset of shear-thinning to lower frequencies. Higher shear thinning is beneficial for injection moulding applications. As shown by Van Puyvelde et al., the rheological behaviour of an immiscible ternary blend is difficult to fully describe considering the numerous potential chemical and physical reactions that are available..$^{50}$ Moreover, in the literature the investigation of ternary blends based on PA6 commonly involves the use of polyolefins (polyethylene, polypropylene) with or without the addition of a compatibilizer. ${ }^{16,51}$ In such ternary blends, despite the wide range of ratios investigated, this shifting of the onset of shear-thinning to lower frequencies has not been observed.

The addition of 5\% PP- $g$-MA in the binary blends dramatically affects the flow of the blends, as shown in Fig. 8c. The complex viscosities of all the blends appear to be higher than the neat polymers and the un-compatibilized blends, across the full range of frequencies investigated. A Newtonian plateau is observed for both the PA6 and PP main phase blends at low frequencies, showing good adhesion between the two compatibilized polymers. Asthana et al. demonstrated through rheological investigation that the maleation of PP decreases the interfacial tension between PA6/PP, inducing a reduction of the particle size of the dispersed phase, as observed earlier by SEM..$^{52}$ After compatibilization, the size of the dispersed phase does not significantly influence the complex viscosity measured. The grafting creates strong chemical bonds between the PA6 and PP-g-MA, as seen in Fig. 3, which will inhibit the independent deformation of the PP and PA6 phases, and as such generate an overall higher viscosity at all frequencies.

Similarly to the binary blend, the addition of a constant concentration of compatibilizer to the ternary blends significantly increases the viscosity of the blends on the range of ratios investigated (Fig. 8d). However, the addition of compatibilizer unfortunately does not markedly influence the PLA depolymerization process. Indeed, the electronegativity of the amine group of PA6 is higher than the carboxylic group of PLA, making the latter less propitious to reactions.

Investigating the linear rheological properties of the blends provides relevant information regarding the flow behaviour of the melts and phase inversion. In Fig. 9, the logarithm of the dynamic modulus $\left(G^{\prime}\right.$ and $\left.G^{\prime \prime}\right)$ is displayed versus the logarithm of the angular frequency at $250^{\circ} \mathrm{C}$ for pure PA6, PP and their (a) uncompatibilized blends and (c) compatibilized blends, and for PA6, PLA and PP ternary blends both (b) uncompatibilized and (d) compatibilized. The storage (or elastic) modulus $G^{\prime}$ is related to the elastic character of the blends or the energy stored during the deformation happening, for instance, during a shearing process. The loss (or viscous) modulus $G^{\prime \prime}$ corresponds to the amount of energy dissipated in the flow under the form of heat or consumed to achieve structural changes into the sample. $G^{\prime}$ corresponds to the sum of the reversible processes, whereas $G^{\prime \prime}$ corresponds to irreversible processes.

An overall observation is that the dynamic modulus of all the blends increases as the frequency increases. Focusing on the storage modulus (square) of the uncompatibilized blends (Fig. 9a), which is particularly sensitive to the morphological state, the blend composition has more effect on $G^{\prime}$ at low 

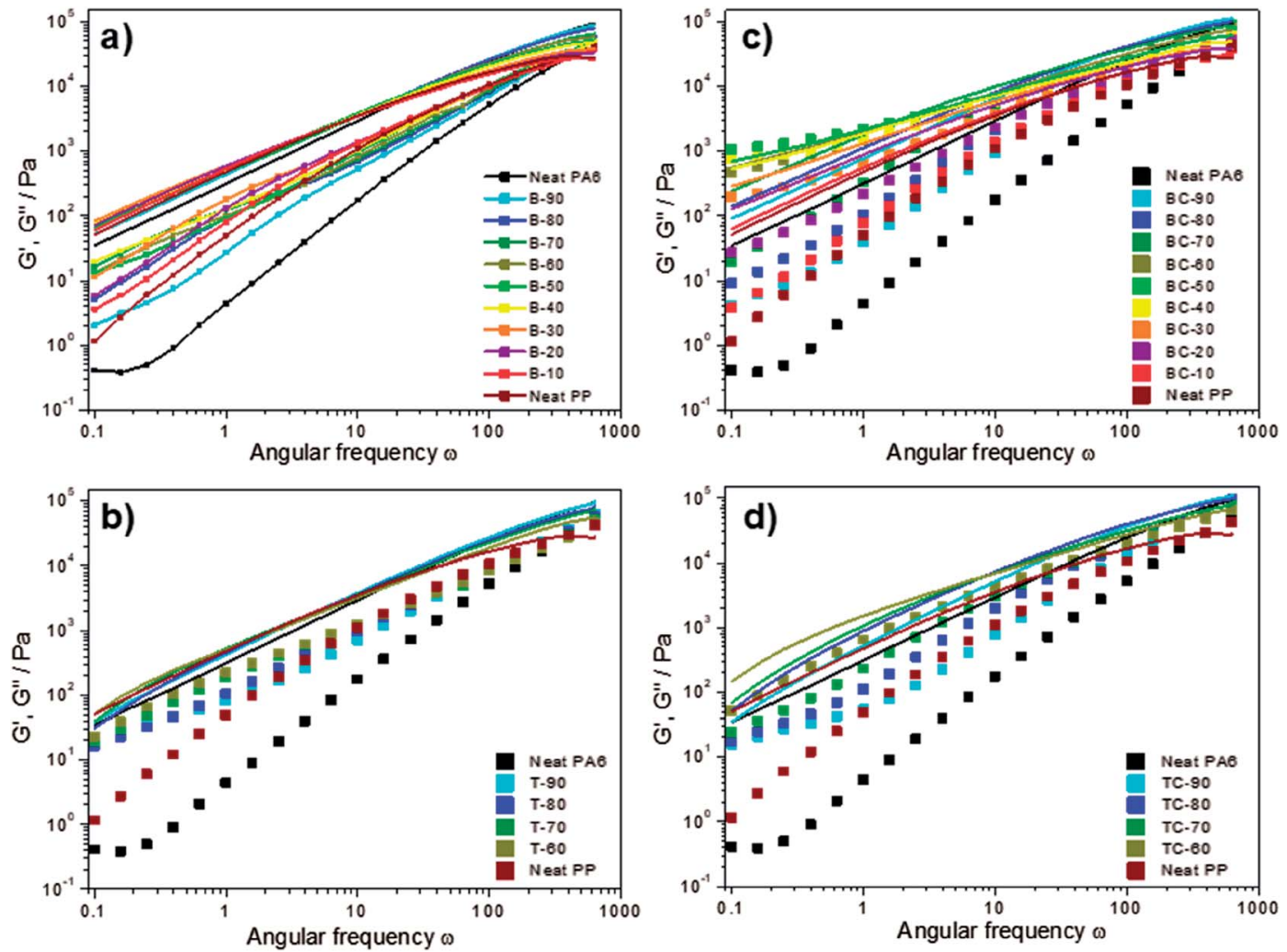

Fig. $9 G^{\prime}$ (square) and $G^{\prime \prime}$ (straight line) versus sweep frequency for binary (PA6/PP) blends (a) un-compatibilized and (c) compatibilized and for ternary (PA6/PLA/PP) blends (b) un-compatibilized and (d) compatibilized, recorded at $250^{\circ} \mathrm{C}$.

frequencies. Similarly to the trend observed in Fig. 8, the storage modulus increases with PP until an optimum was reached with co-continuous phase morphology, before decreasing as PA6 became dispersed. The loss modulus (straight line) of the uncompatibilized blends of different ratios and pure resins did not show any significant difference, meaning that the energy dissipated is almost identical for all the ratios investigated. The complex viscosity variations of the uncompatibilized blends are dominated by $G^{\prime \prime}$, meaning that the melt blends follow a viscous-like behaviour, i.e. mainly influenced by irreversible deformations, typically heat released when the polymer droplets undergo deformations.

After the addition of PLA to the blends, the same trend as obtained in the binary blends was observed. While we could have expected a decrease of the elastic modulus with the PLA fraction increase, due to PLA degradation, the opposite effect was observed. At low frequencies, the elastic modulus of the ternary blend was slightly higher than the binary blend while in the same time the loss modulus was lower. Through the investigation of PLA and polyamide 11 (PA11), Stoclet et al. observed the relatively good self-compatibility of PLA/PA11 blends, which would be a consequence of the relative equivalent polarity of the components. ${ }^{13}$ This result is in accordance with the high interaction obtained previously by interfacial tension measurements and partially explained the particularly small droplets of PLA found in the PA6 matrix.
The addition of $5 \mathrm{wt} \%$ of compatibilizer to these binary blends (Fig. 9c) resulted in significant differences in the rheological behaviour, inherent to the increased interaction between PA6 and PP. Though the dynamic modulus increased when increasing the frequency, the influence of PP-g-MA on $G^{\prime}$ and $G^{\prime \prime}$ shows a simpler behaviour associated with a strong composition dependency. Moreover, unlike the uncompatibilized blends, the same trend is observed for both $G^{\prime}$ and $G^{\prime \prime}$. Indeed, a well-developed dependence of the dynamic modulus on the PP content is observed, and both $G^{\prime}$ and $G^{\prime \prime}$ increase with increase of PP content up to $50 \mathrm{wt} \%$, before decreasing and trending towards neat PP behaviour. A higher elastic modulus is reached for an equivalent weight ratio, meaning that the amount of cooperative motions in the blend polymer chains are higher. Both the grafting and the entanglement density play a role in this behaviour, increasing the overall elasticity of the blends. While the uncompatibilized blends show a dominant loss modulus for every sample and on the full range of frequencies, the compatibilized blends highlight a dominant elastic modulus for intermediate ratios at low frequencies. The cross-links created by the compatibilizer link the phases together, until a blend develops with behaviour that is more elastic than viscous.

The addition of $5 \mathrm{wt} \%$ of compatibilizer to the ternary blend showed a higher elastic modulus, for low PP fractions and TC90 , compared to the binary blends. The TC-90 sample showed a much higher elastic modulus than the BC-90 (Fig. 9c and d). 
The only difference between those two samples is that the PP fraction in BC-90 is replaced with equal content of both PP- $g$-MA and PLA in TC-90. The interaction between PA6, PLA, and PP- $g$ MA appeared to be higher than between PA6, PP, and PP- $g$-MA as highlighted by the higher $G^{\prime}$ values. All of PA6, PLA and PP- $g$ MA are polar components, due to the amine, carboxylic, and anhydride groups respectively in their polymer structure; their closer polarities, in comparison to the apolar PP in polar PA6 and PP- $g$-MA blends, could be a reason for the favoured interactions in the presence of PLA.

The presence of morphological changes in polymer blends can also be revealed unequivocally with Cole-Cole plots. This plot is commonly applied to describe the viscoelastic properties of materials having a high relaxation time distribution, highlighting the phase separation changes. For this, the imaginary viscosity $\left(\eta^{\prime \prime}\right)$ is plotted versus real viscosity $\left(\eta^{\prime}\right)$ yielding to one arc for homogeneous polymer blends, and a tail or even two circular arcs for compositions of different relaxation times. This plot was applied on neat PA6, neat PP, and their binary blends as displayed in Fig. 10a; similarly, the ternary blends were plotted in Fig. 10b. Fig. 10c and d correspond to the homologous blends compatibilized with $5 \mathrm{wt} \%$ PP- $g$-MA.

Several relaxations can be observed on the uncompatibilized curves of binary blends (Fig. 10a). Unsurprisingly, the relaxation process of neat PP shows a single circular arc, highlighting the presence of a homogeneous composition. The relaxation mechanism of neat PA6 shows an arc with a slight tail. An increase of the PP fraction leads to the fast apparition of a second circular arc between 10 and $40 \mathrm{wt} \%$ of PP, highlighting the immiscibility of the PA6/PP blends at all blending ratios. In multiphase blends, Cole-Cole plots generally display two relaxation mechanisms. A first relaxation, corresponding to the high frequencies, is mainly attributed to the intrinsic relaxation of the main component, while at low frequencies relaxations inherent to the deformability of the dispersed phase are occurring. ${ }^{53,54}$ The relaxation of the second arc becomes more obvious with increasing PP concentration. This phenomenon can be explained from a morphological point of view; bigger droplets are more difficult to deform than smaller ones, slowing down the relaxation process. For blends with higher PP fractions (i.e. between 50 and 60\%) the second circular arc leads to a tail, highlighting another change in the relaxation mechanism that we can correlate with the co-continuous morphology observed by SEM.

The addition of PLA to these ternary immiscible blends does not show any extra arc corresponding to the PLA relaxation as we might have expected (see Fig. 10b). Instead, the presence of a steadily increasing line highlights a change in the relaxation mechanism and shows that all the polymers phases are relaxing one after another in a continuous mechanism. In addition, the curves corresponding to the ternary blends were shifted to lower viscosity regions in comparison to the binary blends. The relaxation time corresponding to the ternary blend is slightly lower than the binary probably due to the PLA degradation.
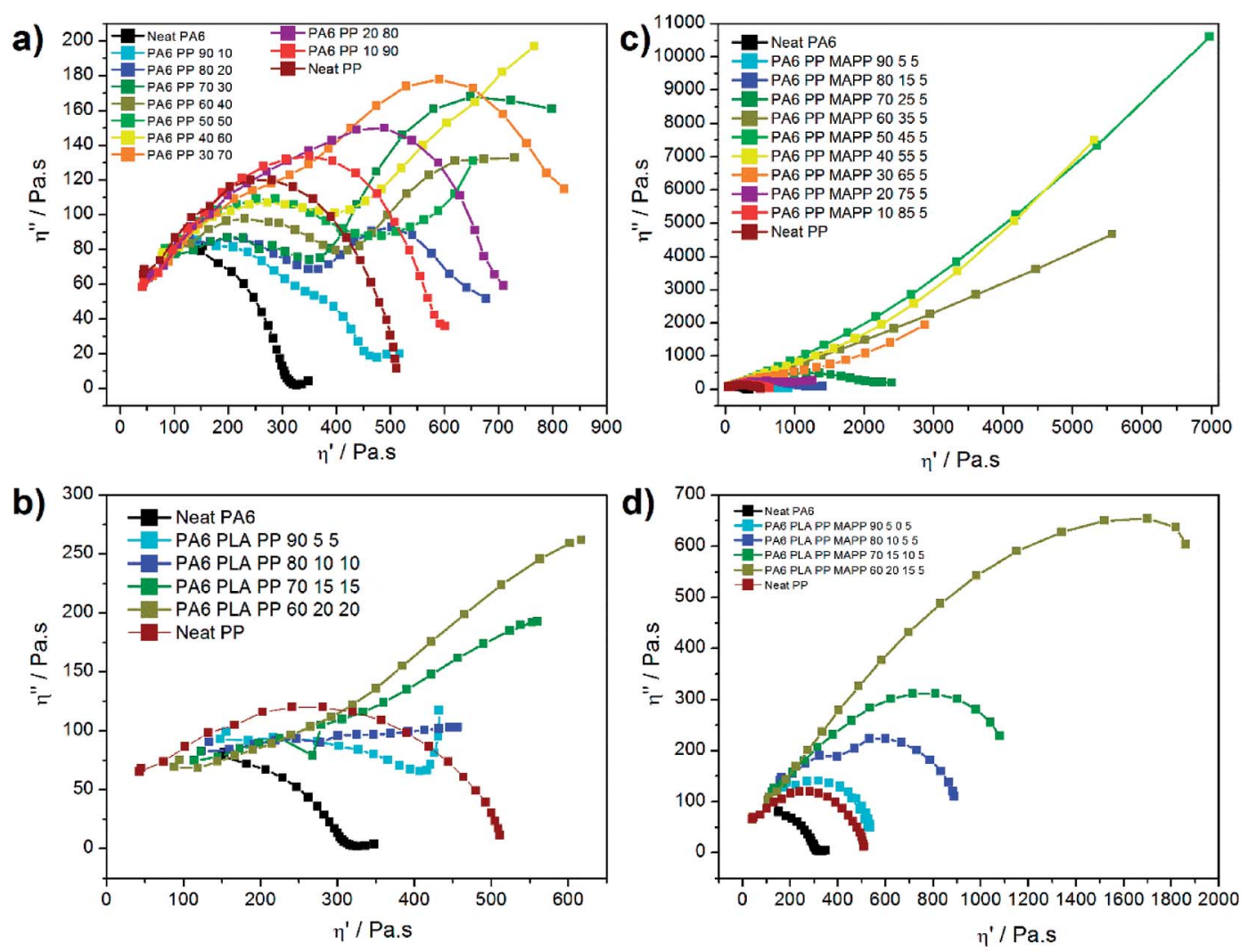

Fig. 10 Cole-Cole plots for neat PA6, neat PP and their binary blends (a) un-compatibilized and (c) compatibilized, and then for PA6-PP-PLA ternary blends (b) uncompatibilized and (d) compatibilized. (The last points of both un-compatibilized and compatibilized ternary blends, corresponding to the polymer degradation, were deleted for the sake of clarity). 
The addition of $5 \mathrm{wt} \%$ of compatibilizer in the binary blends highlights a completely different behaviour as shown in Fig. 10c. The PP-g-MA plays its role perfectly, as only one relaxation is obtained on the full range of ratios, emphasizing the homogeneity of the blends. Moreover, between 35 and $65 \mathrm{wt} \%$ of PP, a second behaviour is observed as shown by the gradual increasing line, which shows similarity to the ternary blends. The material cohesion, as well as the thinner droplets obtained (Fig. 1), delay the relaxation time of the systems. A similar behaviour has already been observed in many compatibilized systems such as PP/EPDM blends compatibilized with maleic anhydride. ${ }^{55}$

After the addition of compatibilizer to the ternary blend, a distinct increase of homogeneity was observed, similarly to the compatibilized binary blend (see Fig. 10d). PP-g-MA appears to compatibilize both PP and PLA phases. Indeed, the ratio TC90 containing only PA6, PLA and PP- $g$-MA shows a single and neat arc stressing the high homogeneity of this blend. The high level of interaction between PA6, PLA and PP is thus highlighted via rheology, for equivalent PA6 content, with a relaxation mechanism much faster than its binary equivalent.

\section{Conclusions}

In this work, the morphology of the PA6/PP/PLA ternary blends was studied and compared to PA6/PP binary blends. After the addition of PLA, a sea-island morphology showing separately dispersed polymer phases was observed by SEM and AFM. Droplets of PLA dispersed phase were significantly smaller than PP dispersed phase, at equivalent weight ratios. Both the interfacial tension and viscoelastic properties of the components enhanced the morphology obtained. The addition of PP- $g$ MA showed a significant reduction of the particle size of PP phase in both the binary and the ternary blends, while the already significantly smaller PLA droplets were not influenced by the addition of compatibilizer. After compatibilization, PP and PLA showed a particular affinity as observed by morphological observations. The morphology observations of the uncompatibilized blends were supported by surface tension measurements and highlighted a higher affinity between PA6/ PLA over both PA6/PP and PLA/PP. While Hobbs' theoretical model and minimum free energy model were applied and pointed to an encapsulation of the PP phase by PLA, no clear confirmation of this morphology could be observed by SEM or AFM investigation, even after etching the PLA phase. This disagreement between theory and observation was explained by the low molecular weight of the PLA phase and its low viscosity in the processing state, resulting in very small PLA droplets and insufficient cohesion to encapsulate the PP phase. Finally, the Cole-Cole plots, obtained from rheology measurements, were applied for all the blends and showed that even a low content of PLA significantly changed the relaxation mechanisms of the ternary blends. The addition of PP- $g$-MA allowed for an increase of homogeneity in all the blends investigated and resulted in an increase of the relaxation times. Rheology experiments verified the high interaction between PLA and PA6 in presence of PP- $g$ MA, as shown by the presence of a single arc on the Cole-Cole plot of the TC-90 blend, emphasizing its good homogeneity. PLA appears to be a good flow enhancer in those blends, and could improve the processability in injection moulding applications.

\section{Conflicts of interest}

There are no conflicts to declare.

\section{Acknowledgements}

This research is financially supported by the Ontario Ministry of Agriculture, Food and Rural Affairs (OMAFRA), Canada/ University of Guelph-Bioeconomy for Industrial Uses Research Program Theme (Project \# 030176); the Natural Sciences and Engineering Research Council (NSERC), Canada - Discovery Grants (Project \# 401111); and the Ontario Research Fund, Research Excellence Program; Round-7 (ORF-RE07) from the Ontario Ministry of Research and Innovation (MRI), currently known as the Ontario Ministry of Research, Innovation and Science (MRIS) (Project \# 052665 and \# 052644).

\section{References}

1 L. A. Utracki and C. A. Wilkie, Commercial Polymer Blends, in Polymer Blends Handbook, Springer, New York, 2014, pp. 1736-1876.

2 C. Harrats, S. Thomas and G. Groeninckx, Micro- and Nanostructured Polymer Blends, 2006, p. 442.

3 C. C. Park, F. Baldessari and L. G. Leal, J. Rheol., 2003, 47, 911-942.

4 T. Huber, M. Misra and A. K. Mohanty, Mechanical properties of compatibilized nylon 6/polypropylene blends; studies of the interfacial behavior through an emulsion model, J. Appl. Polym. Sci., 2014, 131, 9455-9462.

5 Y. Minoura, M. Ueda, S. Mizunuma and M. Oba, The reaction of polypropylene with maleic anhydride, J. Appl. Polym. Sci., 1969, 13, 1625-1640.

6 P. Agrawal, S. I. Oliveira, E. M. Araújo and T. J. A. Melo, Effect of different polypropylenes and compatibilizers on the rheological, mechanical and morphological properties of nylon 6/PP blends, J. Mater. Sci., 2007, 42, 5007-5012.

7 A. González-Montiel, H. Keskkula and D. R. Paul, Morphology of nylon 6/polypropylene blends compatibilized with maleated polypropylene, J. Polym. Sci., Part B: Polym. Phys., 1995, 33, 1751-1767.

8 A. González-Montiel, H. Keskkula and D. R. Paul, Impactmodified nylon 6/polypropylene blends: 1. Morphologyproperty relationships, Polymer, 1995, 36, 4587-4603.

9 G. M. Shashidhara, D. Biswas, B. Shubhalaksmi Pai, A. K. Kadiyala, G. S. Wasim Feroze and M. Ganesh, Effect of PP-g-MAH compatibilizer content in polypropylene/ nylon-6 blends, Polym. Bull., 2009, 63, 147-157.

10 J.-D. Lee and S.-M. Yang, Effects of mixing procedures on properties of compatibilized polypropylene/nylon 6 blends, Polym. Eng. Sci., 1995, 35, 1821-1833. 
11 A. Tedesco, R. V. Barbosa, S. M. B. Nachtigall and R. S. Mauler, Comparative study of PP-MA and PP-GMA as compatibilizing agents on polypropylene/nylon 6 blends, Polym. Test., 2002, 21, 11-15.

12 K. Hamad, M. Kaseem and F. Deri, Rheological and mechanical characterization of poly(lactic acid)/ polypropylene polymer blends, J. Polym. Res., 2011, 18, 1799-1806.

13 G. Stoclet, R. Seguela and J.-M. Lefebvre, Morphology, thermal behavior and mechanical properties of binary blends of compatible biosourced polymers: polylactide/ polyamide 11, Polymer, 2011, 52, 1417-1425.

14 R. Holsti-Miettinen, J. Seppälä and O. T. Ikkala, Effects of compatibilizers on the properties of polyamide/ polypropylene blends, Polym. Eng. Sci., 1992, 32, 868-877.

15 T. S. Omonov, C. Harrats and G. Groeninckx, Co-continuous and encapsulated three phase morphologies in uncompatibilized and reactively compatibilized polyamide 6/polypropylene/polystyrene ternary blends using two reactive precursors, Polymer, 2005, 46, 12322-12336.

16 R. Krache, D. Benachour and P. Pötschke, Binary and ternary blends of polyethylene, polypropylene, and polyamide 6,6: the effect of compatibilization on the morphology and rheology, J. Appl. Polym. Sci., 2004, 94, 1976-1985.

17 D. Wang, Y. Li, X.-M. Xie and B.-H. Guo, Compatibilization and morphology development of immiscible ternary polymer blends, Polymer, 2011, 52, 191-200.

$18 \mathrm{H}$. Li and X. M. Xie, Morphology development and superior mechanical properties of PP/PA6/SEBS ternary blends compatibilized by using a highly efficient multi-phase compatibilizer, Polymer, 2017, 108, 1-10.

19 S. Hobbs, M. Dekkers and V. Watkins, Effect of interfacial forces on polymer blend morphologies, Polymer, 1988, 29, 1598-1602.

20 I. Luzinov, K. Xi, C. Pagnoulle, G. Huynh-Ba and R. Jérôme, Composition effect on the core-shell morphology and mechanical properties of ternary polystyrene/styrenebutadiene rubber/polyethylene blends, Polymer, 1999, 40, 2511-2520.

21 H. F. Guo, S. Packirisamy, N. V. Gvozdic and D. J. Meier, Prediction and manipulation of the phase morphologies of multiphase polymer blends: 1 . Ternary systems, Polymer, 1997, 38, 785-794.

22 M. Hemmati, H. Nazokdast and H. Shariat Panahi, Study on morphology of ternary polymer blends. I. Effects of melt viscosity and interfacial interaction, J. Appl. Polym. Sci., 2001, 82, 1129-1137.

23 T. S. Valera, A. T. Morita and N. R. Demarquette, Study of morphologies of PMMA/PP/PS ternary blends, Macromolecules, 2006, 39, 2663-2675.

24 M. Gao, Z. Ren, S. Yan, J. Sun and X. Chen, An Optical Microscopy Study on the Phase Structure of Poly(L-lactide acid)/Poly(propylene carbonate) Blends, J. Phys. Chem. B, 2012, 116, 9832-9837.

25 J. Reignier, B. D. Favis and M.-C. Heuzey, Factors influencing encapsulation behavior in composite droplet-type polymer blends, Polymer, 2003, 44, 49-59.
26 A. Anstey, A. Codou, M. Misra and A. K. Mohanty, Novel Compatibilized Nylon-Based Ternary Blends with Polypropylene and Poly(lactic acid): Fractionated Crystallization Phenomena and Mechanical Performance, ACS Omega, 2018, 3, 2845-2854.

27 U. A. Handge, K. Hedicke-Höchstötter and V. Altstädt, Composites of polyamide 6 and silicate nanotubes of the mineral halloysite: Influence of molecular weight on thermal, mechanical and rheological properties, Polymer, 2010, 51, 2690-2699.

28 N. Noroozi, L. L. Schafer and S. G. Hatzikiriakos, Thermorheological properties of poly( $\varepsilon$-caprolactone)/ polylactide blends, Polym. Eng. Sci., 2012, 52, 2348-2359.

29 E. E. Ferg and L. L. Bolo, A correlation between the variable melt flow index and the molecular mass distribution of virgin and recycled polypropylene used in the manufacturing of battery cases, Polym. Test., 2013, 32, 1452-1459.

30 R. Tessier, E. Lafranche and P. Krawczak, Development of novel melt-compounded starch-grafted polypropylene/ polypropylene-grafted maleic anhydride/organoclay ternary hybrids, eXPRESS Polym. Lett., 2012, 6, 937-952.

31 S. J. Park, B. K. Kim and H. M. Jeong, Morphological, thermal and rheological properties of the blends polypropylene/ nylon-6, polypropylene/nylon-6/(maleic anhydride-gpolypropylene) and (maleic anhydride-g-polypropylene)/ nylon-6, Eur. Polym. J., 1990, 26, 131-136.

32 C. W. Macosko, H. K. Jeon and T. R. Hoye, Reactions at polymer-polymer interfaces for blend compatibilization, Prog. Polym. Sci., 2005, 30, 939-947.

33 C. A. Orr, J. J. Cernohous, P. Guegan, A. Hirao, H. K. Jeon and C. W. Macosko, Homogeneous reactive coupling of terminally functional polymers, Polymer, 2001, 42, 81718178.

34 D. Godshall, C. White and G. L. Wilkes, Effect of compatibilizer molecular weight and maleic anhydride content on interfacial adhesion of polypropylene-PA6 bicomponent fibers, J. Appl. Polym. Sci., 2001, 80, 130-141.

35 X. Zhou, P. Zhang, X. Jiang and G. Rao, Influence of maleic anhydride grafted polypropylene on the miscibility of polypropylene/polyamide-6 blends using ATR-FTIR mapping, Vib. Spectrosc., 2009, 49, 17-21.

36 T. W. Yoo, H. G. Yoon, S. J. Choi, M. S. Kim, Y. H. Kim and W. N. Kim, Effects of compatibilizers on the mechanical properties and interfacial tension of polypropylene and poly(lactic acid) blends, Macromol. Res., 2010, 18, 583-588.

37 P. Choudhary, S. Mohanty, S. K. Nayak and L. Unnikrishnan, Poly(L-lactide)/polypropylene blends: evaluation of mechanical, thermal, and morphological characteristics, $J$. Appl. Polym. Sci., 2011, 121, 3223-3237.

38 D. K. Owens and R. C. Wendt, Estimation of the surface free energy of polymers, J. Appl. Polym. Sci., 1969, 13, 1741-1747. 39 S. Wu, Polymer interface and adhesion, Marcel Dekker, 1982. $40 \mathrm{~S}$. Wu, Interfacial Energy, Structure, and Adhesion between Polymers, in Polymer blends Volume 1, ed. D. R. Paul SN, Elsevier Science; 1978, pp. 243-293. 
41 D. Wu, L. Yuan, E. Laredo, M. Zhang and W. Zhou, Interfacial properties, viscoelasticity, and thermal behaviors of poly(butylene succinate)/polylactide blend, Ind. Eng. Chem. Res., 2012, 51, 2290-2298.

$42 \mathrm{~S}$. Wu, Predicting chain conformation and entanglement of polymers from chemical structure, Polym. Eng. Sci., 1992, 32, 823-830.

43 L. J. Fetters, D. J. Lohse and W. W. Graessley, Chain dimensions and entanglement spacings in dense macromolecular systems, J. Polym. Sci., Part B: Polym. Phys., 1999, 37, 1023-1033.

44 B. D. Favis and J. P. Califoux, The Effect of Viscosity Ratio on the Morphology of Polypropylene/Polycarbonate Blends During Processing, 1987, pp. 1591-1600.

45 J. R. Dorgan, J. Janzen, M. P. Clayton, S. B. Hait and D. M. Knauss, Melt rheology of variable L-content poly(lactic acid), J. Rheol., 2005, 49, 607-619.

46 S. Mbarek, M. Jaziri, Y. Chalamet and C. Carrot, Effect of the viscosity ratio on the morphology and properties of PET/ HDPE blends with and without compatibilization, J. Appl. Polym. Sci., 2010, 117, 1683-1694.

47 C. D. Han, Rheology of Immiscible Polymer Blends, in Rheology and Processing of Polymeric Materials, Volume 1, Polymer Rheology, 2007.

48 C. Lacroix, M. Grmela and P. J. Carreau, Relationships between rheology and morphology for immiscible molten blends of polypropylene and ethylene copolymers under shear flow, J. Rheol., 1998, 42, 41-62.

49 T. Gerard and T. Budtova, Morphology and molten-state rheology of polylactide and polyhydroxyalkanoate blends, Eur. Polym. J., 2012, 48, 1110-1117.

50 P. P. Van and M. P. Rheology, Morphology Development in Immiscible Polymer Blends, Rheol. Rev., 2005, 101-145.

51 R. Khalili, S. H. Jafari, M. R. Saeb, H. A. Khonakdar, U. Wagenknecht and G. Heinrich, Toward In Situ Compatibilization of Polyolefin Ternary Blends through Morphological Manipulations, Macromol. Mater. Eng., 2014, 299, 1197-1212.

$52 \mathrm{H}$. Asthana and K. Jayaraman, Rheology of Reactively Compatibilized Polymer Blends with Varying Extent of Interfacial Reaction, Macromolecules, 1999, 32, 3412-3419.

53 P. J. Carreau, M. Bousmina and A. Ajji, Rheological Properties of Blends: Facts and Challenges, in Progress in Pacific Polymer Science 3, ed. Ghiggino K. P., Springer, Berlin, Heidelberg, 1994, pp. 25-39.

54 L. Li, B. Yin, Y. Zhou, L. Gong, M. Yang, B. Xie, et al., Characterization of PA6/EPDM-g-MA/HDPE ternary blends: the role of core-shell structure, Polymer, 2012, 53, 3043-3051.

55 P. Ezzati, I. Ghasemi, M. Karrabi and H. Azizi, Rheological Behaviour of PP/EPDM Blend: The Effect of Compatibilization, Iran. Polym. J., 2008, 17, 669-679. 